Accepted refereed manuscript of:

\begin{abstract}
Al Mojamed M \& Kolberg M (2016) Structured Peer-to-Peer Overlay Deployment on MANET: A Survey, Computer Networks, 96 , pp. $29-47$.
\end{abstract}

DOI: $\underline{10.1016 / \text { i.comnet.2015.12.007 }}$

(C) 2016, Elsevier. Licensed under the Creative Commons AttributionNonCommercial-NoDerivatives 4.0 International

http://creativecommons.org/licenses/by-nc-nd/4.0/ 


\section{Structured Peer-to-Peer Overlay Deployment on MANET: A Survey}

\author{
Mohammad Al Mojamed \\ Computing Science and Mathematics \\ University of Stirling \\ Stirling, Scotland \\ mma@cs.stir.ac.uk
}

\author{
Mario Kolberg \\ Computing Science and Mathematics \\ University of Stirling \\ Stirling, Scotland \\ mko@cs.stir.ac.uk
}

\begin{abstract}
There are many common characteristics between Peer-toPeer (P2P) overlay networks and Mobile Ad-hoc Networks (MANET). Self-organization, decentralization, dynamicity and changing topology are the most shared features. Furthermore, when used together, the two approaches complement each other. P2P overlays provide data storage/retrieval functionality, and their routing information can complement that of MANET. MANET provides wireless connectivity between clients without depending on any pre-existing infrastructure. The aim of this paper is to survey current P2P over MANET systems. Specifically, this paper focuses on and investigates structured $\mathrm{P} 2 \mathrm{P}$ over MANET. Overall, more than thirty distinct approaches have been classified into groups and introduced in tables providing a structured overview of the area. The survey addresses the identified approaches in terms of $\mathrm{P} 2 \mathrm{P}$ systems, MANET underlay systems and the performance of the reviewed systems.
\end{abstract}

\section{Keywords}

P2P over MANET, MANET, Cross-Layering, Mobile Ad hoc, Content distribution, Content discovery.

\section{Introduction}

P2P networking paradigms have gained substantial popularity as they support a wide range of applications without the use of centralised servers. P2P networking systems are usually implemented as an overlay network that allows higher-layers communication among participating peers. The established connections between peers are logical and underlay-independent. The initial proposals of P2P systems were mainly targeted at operating in wired networks. However, the very rapid proliferation of wireless communication technology has prompted a need for adoption of $\mathrm{P} 2 \mathrm{P}$ systems in mobile networks too.

On the other hand, MANET are composed of autonomous mobile nodes that communicate wirelessly without relying on pre-existing infrastructure. In such a network, a node acts as a client and a server, similar to P2P overlays. In addition, the participating nodes collaborate with each other through forwarding messages towards other nodes.
There are many common characteristics between P2P overlay networks and MANET. Self-organisation, decentralisation, a dynamic and changing topology are the most important shared features. Crucially, co-implementing MANET with a P2P overlay on top of the physical infrastructure addresses the two issues in MANET: it will help routing decisions in the underlying network and it will provide for data storage. In a MANET nodes may store content locally, or transmit it to a central node for storage. Both approaches pose significant problems: locally stored data is difficult for other nodes to locate, and the central data server requires a nominated node to be maintained and be available. P2P overlay networks have an in-build routing approach to store and locate data items. Another issue in MANET is that the networking layer is inadequate to support sophisticated applications. The mobility of users makes routing challenging. In such networks, routing usually is restricted to level 2 routing based on MAC addresses. Thus P2P overlays complement MANET well.

There is a wide range of systems covered in the literature that adopted structured P2P overlays with MANET. Structured overlays offer a guarantee to locate data items in the network with a maximum number of lookup steps. On the other hand, unstructured overlays organise nodes independently of the stored items. Random walk and flooding are commonly used techniques for retrieval. With an increased network size or an increased number of stored data items, structured overlays outperform unstructured systems. This is due to the significant traffic that unstructured systems may create, causing collision and packet loss in the underlay and thus affecting the system's performance. There are a number of approaches reported in the literature that adapt unstructured systems for use with MANET. However, in order to keep this survey at a manageable size we focus on approaches which combine and integrate MANET with structured, DHT-based, P2P techniques.

A number of related surveys have been published in recent years. Some concentrate on particular aspects of joining P2P and MANET, such as security [1]. Others have a different focus with respect to network protocols used, e.g. focusing on DHT-based routing for MANET [2], concentrating on wireless sensor networks [3] or key management and file sharing [4] [5]. Surveys that are closer in scope to the one 
presented in this paper have been published more than 5 years ago [6][7]. However, a large number of approaches have been published since then. Hence there is a need for a renewed look at recent approaches.

The rest of this paper is divided into six sections. Overviews of P2P and MANET are presented in Sections 2 and 3. Section 4 introduces deploying P2P over MANET and the technical challenges of such deployment. Section 5 covers the literature survey of current P2P systems over Mobile Ad hoc Network and compares approaches in tables, Section 6 discusses the surveyed systems and Section 7 concludes this survey.

\section{Overview of P2P Approaches}

Peer to peer $(\mathrm{P} 2 \mathrm{P})$ networks have attracted a huge interest recently [8][9][10]. The peer to peer communication paradigm enables peers to access distributed resources and services without the need for a centralized server.

P2P networks are self-organizing, adaptable, and highly scalable. P2P networks are offered as an overlay network on top of a physical network. The overlay is responsible for storing and locating services. The overlay provides a peer with the address of another peer that has a copy of the desired content. The peer can then fetch the content through an out of band connection. P2P overlays pose a low level of entry as no major server component needs to be in place. P2P networks can grow or shrink with demand and are highly adaptable.

In terms of topology, decentralized P2P systems can be subdivided into structured and unstructured P2P networks. Early P2P systems used an unstructured topology since they do not follow any particular structure when forming the network. There is no relationship between the stored content and nodes in the overlay. Thus searches are based on Random Walk and Flooding techniques which are inefficient. In fact, no guarantee can be given that a data item which exists in the network can be found by a search. These issues are addressed by introducing structure in the organization of the nodes in the overlay. These overlays are referred to as structured P2P overlays. An overview of such systems is provided below.

Structured P2P networks [11][12] have been devised to address the shortcomings of unstructured systems, namely to provide efficient search strategies that guarantee to reach the destination within a small number of hops. Some systems organize the peers as a multidimensional grid (e.g. CAN [13]), a ring (e.g. Chord [14]), or as a mesh (e.g. Pastry [15]). Structured P2P overlays impose a mapping between a node identifier and the identifier of a data item. Most commonly, such a mapping is achieved through the use of hash functions on the data and node identifiers. Files are located on the peers with the closest hashed node IDs to the hashed file ID. Thus in structured P2P overlays, a data lookup query can be directed towards a particular peer which is responsible for the content required. As a consequence, the searching in the network is much more efficient even if the number of the participating nodes is large. Most of the structured P2P overlays offer a complexity of $\mathrm{O}(\log n)$ for lookups, where $n$ is the number of the participant nodes [12]. However, some systems achieve a $\mathrm{O}(1)$ lookup complexity. This difference in performance is dependent on routing table size and updating mechanisms. A large routing table which is kept up to date through frequent refreshes supports an excellent lookup performance, but comes at the cost of increased bandwidth requirements for the update messages. In addition, an increased level of node churn (nodes joining and leaving the network) requires additional maintenance traffic to keep the data in the nodes' routing tables current.

Consequently, structured overlays can be categorized according to their lookup complexity. Approaches can be split into multi-hop, variable-hop and single-hop overlays.

Multi-hop overlays require multiple hops to deliver a lookup request from source to destination. Well known protocols using this approach are Chord and Pastry. Multi-hop approaches can be further divided into constant degree and logarithmic degree overlays. Logarithmic degree overlays reduce the lookup space in half with each hop. Hence a lookup would require at most $\mathrm{O}(\log n)$ hops, where $n$ is the number of the participating nodes. An example algorithm for this category is Chord. Constant degree overlays require a fixed number of hops to route a message from the originator to the destination. CAN and Cycloid [16] are examples of constant degree overlays.

On the other hand, in one-hop overlays each participating node holds a complete routing table for the whole overlay. If this routing table is fully up to date (which is difficult and costly), only a single hop is needed to achieve routing of a message from source to destination. Examples of such algorithms are OneHop [17][18] and D1HT/EDRA [19].

Variable hops overlays take into account the available bandwidth and adjust their routing table update mechanism accordingly. During times when bandwidth is at a premium, the overlay reduces routing table updates and employs a multi-hop routing technique. However, when bandwidth is more plentiful, the overlay increases its routing table update frequency and employs a one-hop lookup mechanism. Examples variable hop overlays are Accordion [20] and Chameleon [21].

\section{Overview of MANET Systems}

Mobile Ad hoc Networks (MANET) can be defined as collections of autonomous mobile nodes, which communicate wirelessly without any need for pre-existing network infrastructure. In addition, MANET do not rely on centralized control. Each node participating in the MANET is not only regarded as an end system but also as a router 
relaying messages to other participating nodes. MANET are self-configuring and the nodes form a dynamic topology. As the nodes move, they can organise themselves on the fly and hence the topology of MANET may be subject to frequent and unpredictable changes. MANET can be utilized in campus and conference environments, on railways, remote areas without communication infrastructure or areas where the communication infrastructure is down due to natural disaster or political tensions [22]. The number of users in each scenario can range from about 10 nodes in emergency settings, to hundreds of people in a campus or conference setting, to thousands of participants in political unrest or military settings.

A number of surveys of MANET routing have been published. Hong, Xu and Gerla [23] is the oldest but still provides a good overview of the basic concepts. More recent (and more detailed) surveys have been published by Boukerche et al. [24] as well as Alotaibi and Mukerjee [25] Each of these surveys adopts a slightly different categorisation of the approaches. However, for the purposes of this review we will concentrate on the major classes of MANET routing approaches. For a more fine grained classification, please refer to one of the surveys cited above.

Traditionally, MANET routing protocols have been divided into reactive and proactive systems. However, more recently, additional categories of hybrid approaches (using some elements of reactive and proactive systems), hierarchical techniques, geographical routing systems as well as multi-path, multicast and geocast systems have been introduced. Figure 1 shows the main routing algorithm categories used in MANET systems. The following subsections briefly introduce each category and highlight key approaches.

\subsection{Proactive Routing}

In proactive (or table-based) routing [26] each node has routing information for all other nodes in the network. The information is stored in tables in the nodes and maintained by exchanging information with other nodes. Optimized Link State Routing protocol (OLSR) [27] is an example of a proactive routing protocol. Other examples include STAR [28], WRP [29], and QOLSR [30]. Such approaches create a substantial amount of background maintenance traffic to keep all the routing tables up to date. Also the routing tables in the nodes grow as the network size increases. Besides these scalability issues however, proactive approaches have many desirable properties such as low latency route access and QoS path support and monitoring. Proactive routing is best suitable for applications which require on low message latency and which have a high message throughput.

\subsection{Reactive Routing}

Reactive (or on-demand) routing approaches establish a route to the destination node only when needed. Once a route is discovered, it is maintained by the source node until it is no longer required or the route becomes unavailable. Nodes do not maintain a routing table. With reactive routing the route discovery process occurs much more frequently than in proactive routing and the latency for sending a message to a destination is considerably larger due to the initial route discovery process. However, reactive approaches can substantially reduce the control overhead in the network if the frequency of required route discoveries is relatively low. They are best suited for networks with low and medium traffic. In such environments they can be scalable to larger network sizes than proactive approaches. Common examples of reactive routing protocols are Ad hoc On Demand Distance Vector (AODV) [31] and DSR Dynamic Source Routing (DSR) [32][33].

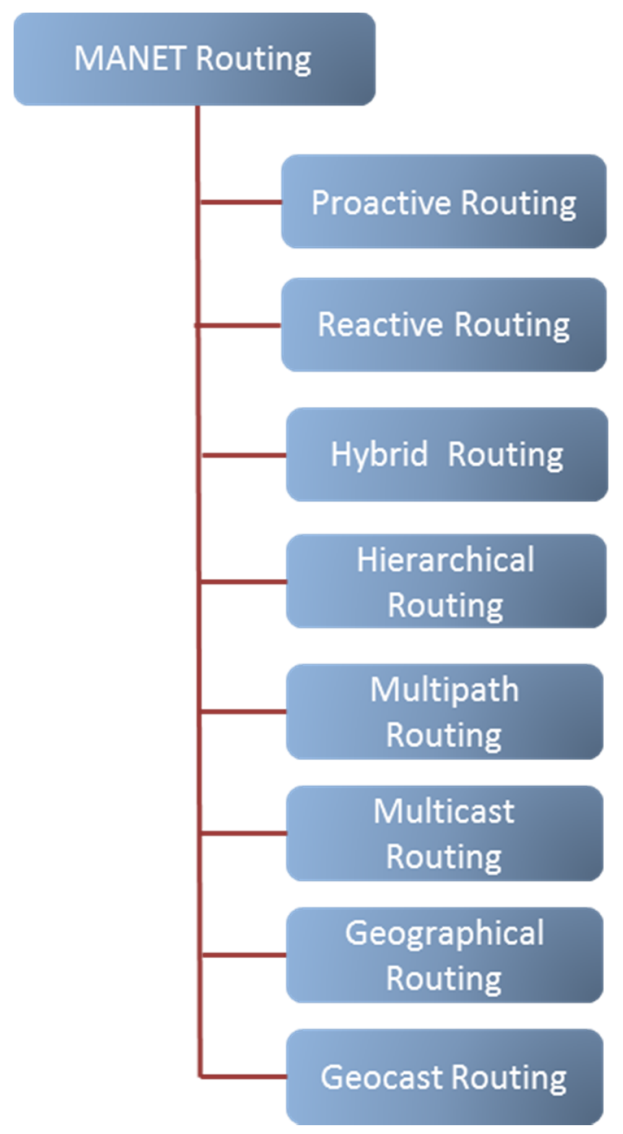

Figure 1: MANET routing systems

\subsection{Hybrid Routing}

Hybrid routing approaches use aspects of both proactive and reactive routing [34]. Commonly, proactive techniques are used to maintain routes to close neighboring nodes, while reactive approaches are used to locate nodes further away. The Zone Routing Protocol (ZRP) [35] is an example of hybrid routing. Hybrid approaches reduce the control traffic required by proactive approaches and also reduce the message latency of reactive approaches. The performance of 
such a network depends on the distribution of two approaches on network nodes. Hybrid approaches can also be implemented using hierarchical network architecture.

\section{$3.4 \quad$ Hierarchical Routing}

Hierarchical routing approaches organise the network nodes into groups called zones or clusters with each group having a head and a gateway. Some surveys differentiate hierarchical and cluster approaches (cluster approaches employ two hierarchy levels whereas hierarchical approaches may support more than two levels). However, for the purpose for this review, the differences are not important and hence we consider these approaches together. While the head is responsible to maintain connectivity between the nodes within the cluster, the gateway manages traffic to and from other clusters. Ordinary nodes can only communicate with their own cluster head (and other nodes within their cluster). Nodes within a cluster are usually either directly connected with the head, or within very few hops. Only gateway nodes can communicate beyond the boundaries of a cluster. Hierarchical routing may implement hybrid routing (as described above). For instance, proactive routing may be used within clusters while reactive routing is used for inter cluster communication. This approach cuts down the maintenance traffic in the network increasing scalability. Hierarchical approaches tend to work well for high-density networks with low node mobility. In networks with high node mobility hierarchical approaches can exhibit low performance due to required changes in the clusters' organisation. Head nodes and gateways pose sensitive points of failure.

\subsection{Multipath Routing}

Multipath routing protocols create multiple distinct paths between a pair of source and destination nodes. Such approaches make better use of the network's resources and more tolerant to network failures than traditional single path approaches. Multipath routing can also make use of the aggregate bandwidth available on parallel paths to achieve better throughput. This is especially useful for highbandwidth transmissions. Multipath approaches can avoid network bottlenecks and achieve redundancy in the message transmission to the destination node. However, all these advantages come at a cost: a potentially substantially increased complexity in the route discovery process as disjoint routes between pairs of nodes are required.

\subsection{Multicast Approaches}

Multicast routing approaches transmit a packet to a set of destination nodes. There are tree-based, mesh-based, and group forwarding based as well as hybrid multicast protocols [36]. Most MANET multicast approaches are treebased creating a tree from the source to the destinations. An example of tree based multicasting is Ad hoc Multicast Routing (AMRoute) [37]. The mesh-based network consists of set of interconnected nodes between which a message can be sent. It is seen as a more robust alternative to tree-based approaches since backup paths are available in the event of a path failure. Core-Assisted Mesh Protocol (CAMP) is an example [38]. With group forwarding-based approaches a set of nodes are chosen to be the forwarding nodes being responsible for forwarding of multicast messages. Such approaches are also termed core based approaches and may take into account the state of the underlying network. Core nodes may be high performance nodes or nodes with additional resources. On-demand multicasting routing protocol (ODMRP) is a group forwarding multicast protocol [39]. Hybrid multicast approaches combine both tree-based and mesh-based techniques. Efficient Hybrid Multicast Routing Protocol (EHMRP) [40] is an example of this type of protocol.

\subsection{Geographical Routing approaches}

With geographical routing approaches, a sender uses the geographical location of a destination to route the message. Thus the node's location is used for routing rather than its network address. The main advantage of these approaches is that nodes do not need full knowledge of the network topology. However, each node needs to be aware of its own location, and a source node needs to know the location of the destination node. The Global Positioning System (GPS) is typically used to work out a nodes location. However, sometimes, a node is unable to establish its exact location (for instance inside buildings). Thus the assumption that each node knows its location may not be achievable in certain conditions.

\subsection{Geocast Approaches}

Geocast approaches are sending a message from a source node to a group of destination nodes based on their geographic location. Thus in geocast routing the physical location of destination nodes determines group membership, that is, nodes in a specific geographic location belong to the same group. Geocast approaches are a merger of geographic routing and multicast techniques. Destinations may be defined as different shapes, such as point, circle or polygon. Location-based Multicast (LBM) [41] and GeoGRID [42] are examples of geocast routing protocols.

\section{Deploying P2P Overlays on MANET}

\subsection{Challenges}

Deploying P2P overlay networks on MANET faces a number of challenging issues [6][43]. The following list summarises the key challenges:

Limited bandwidth: This is one of the most fundamental challenges when deploying P2P over MANET. Current P2P overlay algorithms can be quite wasteful with bandwidth. However, MANET bandwidth resources are more constraint when compared with a wired IP infrastructure. As a result, deploying standard P2P algorithms will incur high 
maintenance overhead and hence are unsuitable for MANET.

Logical overlay maintenance: In order to maintain their routing tables, DHT protocols periodically send maintenance requests and responses to learn about unavailable peers. This traffic duplicates route discovery in MANET and hence is undesirable.

Physical topology changes: By their nature, MANET nodes are mobile which can break links between nodes and hence has an impact on the overlay. Thus, in order to have a consistent overlay, the P2P system needs to be informed about the changes in the underlay. Clearly, this incurs a cost. Routing stretch: In a system combining P2P overlay and MANET underlay, routing is implemented both at the physical (MANET) layer and the logical (P2P) layer. Crucially, each hop in the overlay corresponds to a path in the underlay. Furthermore, two nodes which are close in the overlay, may be far apart in the MANET, and nodes which are physically close may be multiple logical hops apart. Routing stretch is the ratio between the logical and the optimal physical path. As a result of this a lookup message may require a number of logical hops in order to access a physically close service. Hence minimising routing stretch is one of the most critical challenges.

Network resiliency: When a peer fails in the structured P2P overlay, the overlay will adapt and discover an alternative path. The same mechanism can be applied at the MANET layer when used in conjunction with an overlay. However, operating such mechanisms in MANET is expensive. Thus network resiliency is an important issue.

Query propagation: Due to the fact a direct hop in the overlay translates to a potential long path in the underlay, a high frequency of overlay messages may lead to path congestion in the underlay. Clearly this should be reduced as much as possible. Approaches include limiting the query range through using TTL and using smart relaying (relaying node waits for a period before rebroadcasting the message).

Battery power: As most $\mathrm{P} 2 \mathrm{P}$ overlay algorithms were designed for a wired environment, they do not take into account the constraints imposed by limited battery power availability. However, when using such algorithms with MANET, the number of messages needs to be minimised to conserve battery power.

Infrastructure-less: the lack of infrastructure with MANET makes it difficult for some P2P protocols to be adopted for use with MANET. For instance, CAN relies on using static landmarks when assigning logical IDs.

\subsection{Deployment Approaches}

MANET and P2P overlays traditionally operate at different layers in the protocol stack. P2P overlays reside and operate at the application layer while the MANET protocols are concerned with connectivity between nodes and work at the network layer. In order to deploy P2P overlays over MANET there are three possibilities:

\subsubsection{Legacy design}

The most straightforward design is to build a P2P overlay on top of the network layer. However, simply adding a P2P overlay on top of MANET is not a functional solution. Many of the issues outlined above will make such a system almost unworkable. Such a system would be a highly inefficient solution as both layers will operate their own routing algorithms to maintain connectivity between logical and physical neighbours. This results in considerable redundancy and very poor performance. Figure 2 depicts this approach. Such a system does not exploit any synergies which exist between the overlay and underlay routing. Still a number of systems following this approach have been experimented with. Backtracking Chord [44] uses modified Chord with AODV.

\begin{tabular}{|c|c|}
\hline P2P Protocol & $\begin{array}{c}\text { Application } \\
\text { Layer }\end{array}$ \\
\hline MANET Routing Protocol & $\begin{array}{c}\text { Network } \\
\text { Layer }\end{array}$ \\
\hline IEEE 802.11 & $\begin{array}{c}\text { MAC } \\
\text { Layer }\end{array}$ \\
\hline Network Interface & $\begin{array}{c}\text { Physical } \\
\text { Layer }\end{array}$ \\
\hline
\end{tabular}

Figure 2: Layered Design for using P2P on MANET.

\subsubsection{Cross-Layer design}

In order to improve the performance offered by the layered design, P2P overlays need to know about the status of the underlay network. This would help reducing the maintenance overhead and thus result in a better overall performance. On the other hand, MANET algorithms should also be aware of the P2P overlay in order to maintain the appropriate connections with relevant neighbouring overlay nodes. This type of approach is termed a Cross-Layer design. However, this design violates the layered architecture since it allows sharing information among nonadjacent layers. Information at the network layer is made available to the application layer and vice versa. However, permitting such an exchange of information has the potential to reduce the incurred overhead considerably. Figure 3 depicts the cross-layer design. CrossROAD is a well-known example that follows this approach by allowing Pastry to communicate with AODV [45].

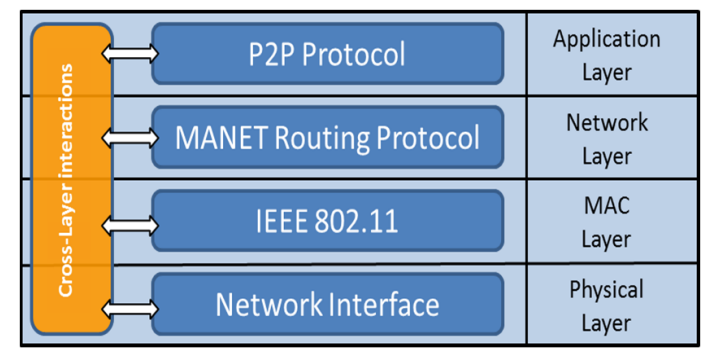

Figure 3: Cross-layer Design. 


\subsubsection{Integrated Design}

The third possibility to deploy P2P over MANET is to integrate the P2P algorithms directly with the network layer. Clearly, such a close integration eliminates redundant routing between the two layers at the same host. However, while this is another form of violating the layered architecture, it leads to an improved performance. Figure 4 shows this design option. Ekta [46] is an example that belongs in this category. Ekta integrates Pastry with DSR into one structure to exploit the synergies between the two algorithms for an improved routing performance.

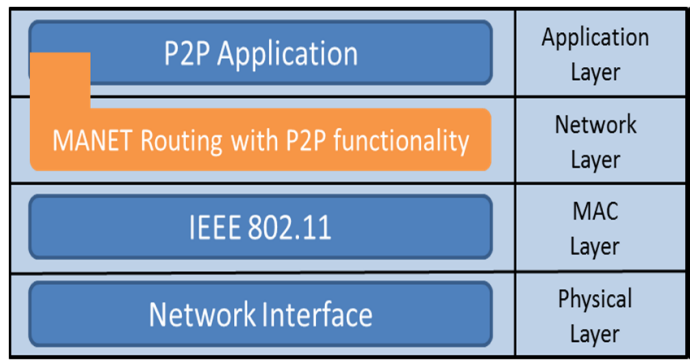

Figure 4: Integration Design.

\section{Categories of Systems integrating Structured P2P with MANET}

\subsection{Methodology of the Survey}

P2P technology in MANET networks can be categorised in a number of different ways. Firstly, they can be categorised according to their integration design (see Section 4.2). Secondly, they can be categorised according to the type of P2P overlay used and thirdly they may be categorised according to the purpose of employing the distributed hash table. Considering the latter, two main groups of systems can be distinguished. There are systems that have used the DHT to enhance the performance of MANET routing whereas others use the DHT for improved data storage and retrieval.

If the used $\mathrm{P} 2 \mathrm{P}$ overlay is the base for categorisation, at the most basic level approaches which use unstructured and structured overlays can be distinguished [47]. As this paper focusses on approaches employing structured overlays, such approaches can be further divided into subcategories according to the hop-count performance of the overlay algorithm (one-hop, multi-hop, variable hop).

Systems can also be classed according to the used underlay algorithms. The following categories can be identified: proactive underlay based systems, reactive underlay based systems, GPS-based systems and finally underlay independent systems. Some systems which use a GPS-based approach may also employ a proactive or reactive underlay. In this review such an approach would be classed as a GPSbased technique as this is a more specific feature than the type of underlay used. Thus approaches which are listed as proactive or reactive-based do not fit in another category, whereas GPS based systems may also be classed as proactive or reactive systems. The tables in the sections provide further details.

For this survey the purpose of the system is used as the top level categorisation. Furthermore, data storage systems are further categorised according to the underlay system used. On the other hand, systems that have used P2P algorithms to enhance MANET routing can be subdivided according to the structure of the adopted P2P technique. There are RingBased systems, Tree-Based systems and systems built on other structures. These categories are also used to structure the remainder of this section. Section 5.2 focusses on approaches that adopted P2P functionality to support data dissemination in MANET. It introduces the aforementioned four subcategories; proactive underlay based systems, reactive underlay based systems, GPS based systems and independent of the underlay systems. Section 5.3 covers the second main category; approaches that have employed P2P technology to improve routing in MANET networks. In total, more than thirty approaches have been categorised in this review.

There are also a limited number of systems which discuss optimisations of the P2P overlay for use on mobile networks. Key concerns for such approaches are conserving battery power or bandwidth usage. Networks where such approaches are applicable include the mobile phone data network (e.g. 3G), large scale sensor networks or even Wifi networks. As these approaches are not specifically targeted at MANET, we do not consider these in this review.

Figure 5 shows the classification of structured P2P over MANET as used in this survey.

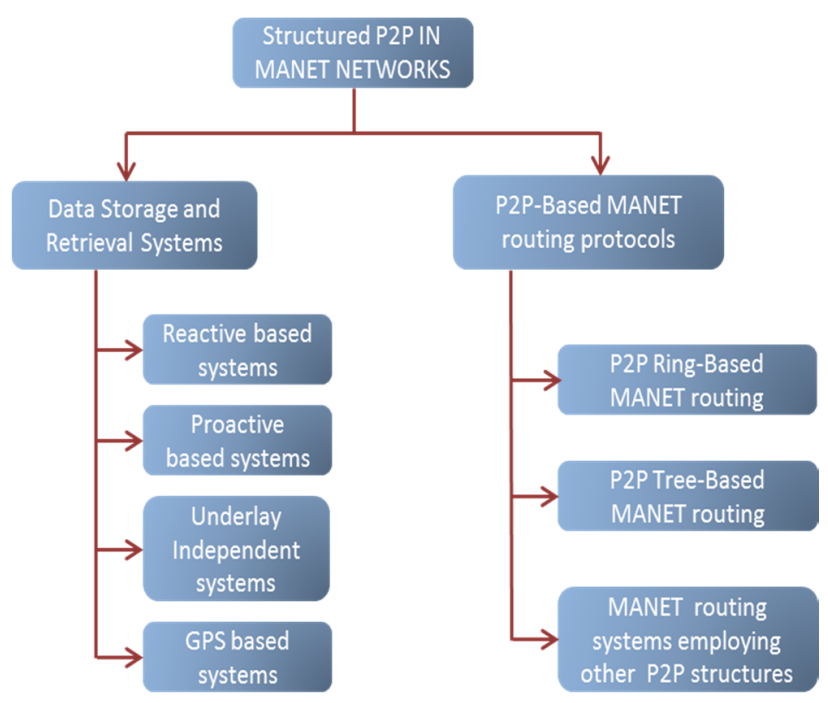

Figure 5: Structured P2P in MANET 


\subsection{P2P overlays over MANET}

\subsubsection{Proactive-based systems}

Proactive routing systems maintain routing information to other nodes in the network in advance of their use. The proactive underlay permits optimization of the $\mathrm{P} 2 \mathrm{P}$ overlay as the underlay provides complete information of the routes in the mobile network. Commonly, the generated traffic in pro-active based networks is also less than systems using a reactive underlay. This is due to the fact that $\mathrm{P} 2 \mathrm{P}$ overlays frequently send messages between any two nodes in the network. That means in a reactive system, route discovery requests are very common. Indeed they are so common, that reactive systems lose their advantage over proactive systems of lower bandwidth consumption. This is especially true in systems with high node mobility. For instance, Shah et al. [48]and Abid et al. [49] claim that with a proactive underlay, a lookup issued by a peer is immediately transmitted to the target node using the shortest underlay path. This helps eliminate route discovery overhead used by reactive protocols and leads to relatively short latency. In addition, proactive underlay suit networks with high node mobility and intensive lookup traffic. Reactive based systems are easily overloaded in such settings. Abid et al. use OLSR to calculate the weight of the links between peers. The approach builds a graph at each node and calculates the weight of each link in terms of the physical hops involved. Systems that use proactive underlays are CrossROAD [50], Shah et al. [51], MA-SP2P [48], P2P MMOG over MANET [52], Chord in Mobile ad hoc network [53], 3DO [49], and ROBUST [54] [55]. All of these systems have used OLSR as the underlay routing protocol.

CrossROAD [50] have adapted Pastry to operate over OLSR. In CrossROAD, each node maintains a global services table that stores all the services provided by all the nodes in the network. It uses an External Data Sharing Module (network status [45]) to allow cross-layering between overlay and network layer. Services are updated through cross layering with the underlay where the proactive flooding of OLSR is optimized to serve the Service Discovery protocol. When a new CrossROAD peer wants to join the overlay, it subscribes first with the desired service from the service repository. It can then retrieve information about peers that provide this service and build its view of the overlay. CrossROAD peer maintains a logical routing table organised similarly to the prefix based routing employed by Pastry.

Shah et al., MA-SP2P and 3DO adopt a similar structure. They construct a minimum spanning tree (MST) to build an overlay that better matches the physical underlay. Each peer builds a graph that includes the peer itself and nodes which are up to 2 logical hops away. The graph is then used to construct the MST. Shah et al. use a root peer to connect all peers in the network and to be used as a reference point. As a reference point, the root peer will be consulted whenever peers build a neighbour relationship. The root peer announces one of them to be in charge of maintaining the relationship. The chosen peer will send frequent probe messages to its neighbour. Each peer maintains a disjoint portion of the ID space. The ID space is distributed between peers so that a peer $\mathrm{P}$ has a connected neighboring peer $\mathrm{P} 1$ with the lower end value of the P's portion of the ID space being greater than the upper end value of $\mathrm{P} 1$ 's portion. Each peer is required to maintain a routing table that stores information about the root peer and neighbor peers including their disjoint portion of the ID space. During routing, a query is sent to one of the directly connected neighbors whose portion of the logical space is closest to the destination. This approach will be repeated until the destination is found.

MA-SP2P is an extension of the previous system. It does not use a root peer. Each peer in MA-SP2P maintains a disjoint portion of the ID space that may be nonconsecutive. A data item or key is stored on a peer P if the hash value of the key falls in the P's portion of the ID space. MA-SP2P distributes the ID space in a way that the Lower End (LE) value of a portion at peer $\mathrm{P}$ points to neighbour peer P1 that has a portion of the ID space lower than LE. In a similar way, the Upper End (UE) of a continuous portion at $\mathrm{P}$ should point to a neighbour $\mathrm{P} 2$ that has a portion with a greater value of ID space. MA-SP2P peers use frequent probe messages which are exchanged between directly connected neighbours to maintain the overlay.

3DO attempts to solve the mismatch problem between the underlay and the overlay topologies through interpreting the exact physical relationships between nodes at the logical level. It considers the relationships between neighbouring peers when computing a peer's logical ID. Peers view the logical address space as a 3D rectangular coordinate system when computing logical IDs. Each 3DO peer is assigned a transient logical identifier corresponding to its relative location in the 3D overlay. The identifiers take the form of a three tuple $\{x|y| z\}$. For each axis, the identifier ranges from 1 to $\pm 2^{m}-1$. Probe messages are exchanged between neighbours periodically to maintain the overlay. 3DO assigns weights to the links between peers corresponding to the number of physical hops between them. This information is gathered from the underlay protocol (OLSR).

P2P MMOG targets Massively Multi-player Online Games (MMOG) over MANET. It uses clustering and landmarking in a similar fashion to MADPastry. It divides the network into clusters with peers sharing a common overlay ID. It organises peers in a two-level hierarchical structure; a local DHT and a remote DHT. Each of the clusters has a head who exchanges information on its cluster with neighbouring cluster heads. The global DHT is used to locate the cluster heads following a prefix strategy as in Pastry. The local cluster is further organised as a Virtual Ring. Each peer in the network is assigned a hierarchical identifier which is used for routing purposes. 
Table 1: Proactive-based systems

\begin{tabular}{|c|c|c|c|c|c|}
\hline $\begin{array}{c}\text { System Name, } \\
\text { Authors, Reference }\end{array}$ & P2P system & MANET Routing & $\begin{array}{l}\text { Deployment } \\
\text { method }\end{array}$ & $\begin{array}{l}\text { Mobility Model and } \\
\text { Max Node Speed }\end{array}$ & Simulation/Evaluation \\
\hline $\begin{array}{c}\text { CrossROAD, } \\
\text { Delmas tro et al. [50], } \\
2005\end{array}$ & Pastry & OLSR & Cross-layer & No & $\begin{array}{l}\text { Testbed was used to evaluate the } \\
\text { performance of CrossROAD using } 8 \\
\text { nodes. }\end{array}$ \\
\hline $\begin{array}{l}\text { Shah and Qian } \\
\text { [51], } 2010\end{array}$ & $\begin{array}{c}\text { Structured P2P, } \\
\text { MST }\end{array}$ & OLSR & Layered & $\begin{array}{l}\text { Random Way Point, } \\
0.4,0.8,1.2,1.6 \text {, and } 2 \\
\mathrm{~m} / \mathrm{s} \text {. }\end{array}$ & $\begin{array}{l}\text { Compared to Da Hora et al [56]. } 100 \\
\text { nodes. }\end{array}$ \\
\hline $\begin{array}{c}\text { MA-SP2P, Shah, } \\
\text { Qian, Wang [48], } 2012\end{array}$ & $\begin{array}{c}\text { Structured P2P, } \\
\text { MST }\end{array}$ & OLSR & Cross-layer & $\begin{array}{c}\text { Random Way Point, } \\
0.4,0.8,1.2 \text {, and } 1.6 \\
\mathrm{~m} / \mathrm{s}\end{array}$ & $\begin{array}{l}\text { Compared to modified Chord over } \\
\text { AODV, Shah etal.[2010] and Hashline. } \\
100 \text { nodes. }\end{array}$ \\
\hline $\begin{array}{l}\text { P2P MMOG over } \\
\text { MANET, Yu and } \\
\text { Vuong [52], } 2011\end{array}$ & $\begin{array}{l}\text { Structured } \\
\text { hierarchical } \\
\text { overlay }\end{array}$ & OLSR & Integration & No & No evaluation \\
\hline $\begin{array}{c}\text { Cramer, Fuhrmann } \\
\text { [53], } 2006\end{array}$ & Chord & OLSR & Layered & $\begin{array}{l}\text { Random Direction } \\
\text { Model. Speed: } 0,2 \text {, } \\
\text { and } 5 \mathrm{~m} / \mathrm{s}\end{array}$ & $\begin{array}{l}\text { For comparison, other flood-based } \\
\text { protocol was implemented. It was also } \\
\text { compared to Chord layered over DSR } \\
\text { and AODV. }\end{array}$ \\
\hline $\begin{array}{l}\text { 3DO, Abid, Othman, } \\
\text { Shah, [49], } 2014\end{array}$ & $\begin{array}{c}\text { 3D rectangular } \\
\text { coordinate } \\
\text { system }\end{array}$ & OLSR & Cross-layer & $\begin{array}{l}\text { Random Way Point } \\
0.5 .1,1.5 \text { and } 2 \mathrm{~m} / \mathrm{s} \text {. }\end{array}$ & Compared to MA-SP2P. \\
\hline $\begin{array}{l}\text { ROBUST, Millar et } \\
\text { al. [54][55], } 2012\end{array}$ & Bamboo & OLSR & layered & Speed: $1 \mathrm{~m} / \mathrm{s}$ & $\begin{array}{l}\text { compared to Bamboo over MANET, size } \\
\text { of network varied from } 20 \text { to } 70 \text { with only } \\
\text { up to } 50 \% \text { of them are moving. }\end{array}$ \\
\hline
\end{tabular}

Cramer and Fuhrmann [53] implement Chord over a number of underlays including a proactive system to investigate the issues of deploying P2P over MANET. This was a layered deployment of Chord over MANET with no protocol changes or optimisations. Their conclusion was that the poor performance of Chord over MANET was not a result of congestion. It is a result of the protocol failover strategy where it assumes the destination has left the network when packet gets lost.

ROBUST by Millar et al. $[54,55]$ is based on the structured P2P system Bamboo. However, it distinguishes itself from Bamboo by introducing hierarchical clustering of the peers. Peers are clustered corresponding to their proximity in the underlay. Each cluster has a super peer, which is in charge of its cluster members. In addition, cluster super peers are also responsible for cluster maintenance. Each cluster is restricted to a specific size and all of its peer members are a single logical hop away from the cluster super peer. Each ROBUST peer is assigned a logical identifier which is related to the ID of their super peer. Beacon packets are sent periodically by the super peer to all of its members. Member peers are expected to forward these packets with $\mathrm{TTL}=1$ to allow other peers from different clusters to detect if they are moving closer to this super peer. If a peer is closer to a new cluster, it requests a new ID from the new super peer. Table 1 summarises proactive-based systems.

\subsubsection{Reactive-based systems}

This group of approaches has proposed P2P systems for MANET using reactive MANET. MANET routing protocols employed includes the Ad hoc On-demand Distance Vector (AODV) and Dynamic Source Routing (DSR). Both protocols work on demand and discover routes as they are needed. Therefore, low levels of traffic are generated when there is less demand from the overlay. However, structured overlays usually require a base level of maintenance traffic which results in additional routing traffic at the underlay. Thus, in scenarios with high node mobility and high overlay maintenance traffic, the network may be overwhelmed with a reactive underlay. The main reason for this is the route discovery and maintenance procedures of reactive MANET routing protocols. Furthermore, overlay messages encounter higher latency which clearly affects the performance of the overlay. However, in the case of little or no node mobility and with a low maintenance overlay system, reactive underlay routing has the advantage of reducing the amount of MANET traffic. AODV is claimed to give the best efficiency when deploying P2P over MANET [56]. Systems that use reactive MANET routing protocols are Hashline [57], Backtracking and Redundant Chord [44], MRDP [58], Bamboo/AODV [59], Convergence Chord [60], Chord in mobile ad hoc network [53] and da Hora et al.[56]. Table 2 lists these systems. 
Hashline builds a one dimensional logical space like a line and hashes keys to their corresponding points on the line. The responsibility of the keys is distributed between peers in the logical line structure with a Hashline peer is in charge of a segment of the line. To determine the logical route between nodes, Hashline optimise a tree-structure to help in accessing a required node. This structure is built from the graph that represents the connectivity in the physical topology. Thus, adjacent peers in the overlay are also close to each other in the underlay. When a new peer joins the overlay, it receives a portion of the Hashline from its closest peer in the physical underlay. Afterwards, the joining peer takes responsibility of this part of the Hashline. Furthermore, the joining peer considers the peer, from which it receives part of the Hashline, as its parent in the logical tree structure. The joining peer will also be added as one of the parent node's children. In order to find a key, a peer sends the request to its parent. The parent checks if it has the requested key or one of its children has it. Otherwise, the request is forwarded to the parent's parent after adding the current parent to the route list of the request.

Backtracking and Redundant Chord [44] has introduced some modification to Chord to enhance its performance in MANET. The modified versions of Chord are deployed over AODV as the MANET underlay. Two approaches, Backtracking and Redundant Chord are proposed. The former sets a timer for a query search. If it times out before receiving any reply, a new query is sent to another successor. The timeout value is set between 0 and $\log \mathrm{N}$, where $\mathrm{N}$ is the number of peers in the network. While, Backtracking Chord may increase the success rate of Chord in MANET, it does so at the cost of higher lookup latencies. Backtracking Chord may increase the success rate of Chord when used with MANET but at a cost of a higher delay to achieve lookup response. On the other hand, Redundant Chord sends $r$ queries to $r$ successors concurrently aiming to reduce the latency when looking up an object in the network. $\mathrm{R}$ is set between 0 and $\log \mathrm{N}$. Redundant queries may overload the network.

MRDP integrates a DHT with AODV at the network layer. The authors claim that the DHT is only used for caching information and not for MANET routing purposes. MRDP forms nodes into dynamic clusters. It utilizes the DHT to cache information and search for resources in each cluster. When the desired object is not found in the same cluster, broadcasting starting from the originator is used to locate it elsewhere. Once the resource is found, the originator of the request sends a publish request to its cluster head. The aim of this is to cache information about the resource within the local cluster. MRDP requires a geometric relation between a cluster head and its members. Cluster members should be physically close to the cluster head and are n-hops away from it. Cluster heads are selected according to grades. An example is the link quality of the mobile node. Consequently, nodes with a high quality link are chosen as cluster heads. In networks with low node mobility, the grade may also be the degree of the node. Thus, nodes with a large number of neighbours are selected as cluster heads. For cluster maintenance, MRDP adopts a proactive strategy where each node periodically broadcasts Hello messages with TTL $=1$. Such messages contain information such as network grade, neighbours and cluster head.

Bamboo/AODV [59] layers Bamboo over AODV. They evaluated different settings to find a good balance between overlay management traffic and network congestion. Bamboo operates a proactive management strategy to maintain connectivity between overlay peers. Peers exchange periodic maintenance messages. Bamboo/AODV has examined three types of management; No management, standard management and custom management. The latter was the recommended approach for Bamboo when deployed over MANET. It is claimed that with the custom setting, the protocol can achieve its best performance in terms of overhead traffic and lookup efficiency. In the custom setting, the frequency of local routing and global routing update were almost doubled and the data storage update was tripled

Convergence Chord [60] has been proposed to solve convergence problem for separated Chord rings in MANET. It exploits routing layer information through cross layering to detect any neighbouring node that belongs to another Chord ring. In addition to Chord routing tables, it maintains another table to detect ring separation called Chord Neighbour Table (CNT) which stores information about physical neighbours. Updates for this table come from the underlay routing protocol. The overlay peer then hashes the received IP addresses and stores them in the CNT tagging them with 0 for a new neighbour that should be identified and 1 for an already known neighbour.

Cramer and Fuhrmann [53] implement Chord over two different reactive underlays including DSR and AODV. Their aim was to investigate the issues of deploying P2P over MANET. They conclude that the problem of deploying Chord over MANET is not the resulted overhead from the overlay but the protocol's pessimistic timeout and failover strategy.

Da Hora [56] has also introduced some modification to Chord to suit MANET and layered the proposed system over AODV. Da Hora claims that the poor performance of Chord over MANET is due to the loss of query messages. Similar to Redundant Chord [44], Da Hora modified Chord so that the originator sends the query to the $n$ closest entries in the finger table rather than just to a single peer. This modification applies only to the query initiating node. The objective of introducing redundancy is to increase the probability of the query to be solved. The receiver of the query follows the traditional Chord algorithm. Da Hora et al. conclude that there is trade-off between success rate, energy consumption and the number of redundancy. 
Table 2: Reactive-based systems

\begin{tabular}{|c|c|c|c|c|c|}
\hline $\begin{array}{c}\text { System Name, } \\
\text { Authors, Reference }\end{array}$ & P2P system & MANET Routing & $\begin{array}{l}\text { Deployment } \\
\text { method }\end{array}$ & $\begin{array}{l}\text { Mobility Model and } \\
\text { MaxNode Speed }\end{array}$ & Simulation/Evaluation \\
\hline $\begin{array}{c}\text { Hashline, } \\
\text { Sozer, Tekkalmaz, } \\
\text { Korpeoglu } \quad[57], 2009\end{array}$ & $\begin{array}{l}\text { Structured one- } \\
\text { dimensional } \\
\text { space. Similar } \\
\text { to CAN. }\end{array}$ & DSR & cross-layer & $\begin{array}{c}\text { Random Way Point } \\
0.1,0.2,0.3,0.4,0.5 \\
0.6,0.7,0.8,0.9,1 \\
\mathrm{~m} / \mathrm{s} .\end{array}$ & $\begin{array}{l}\text { Compared to flooded approach. No. of } \\
\text { nodes varies between } 10 \text { and } 100 .\end{array}$ \\
\hline $\begin{array}{c}\text { Backtracking and } \\
\text { Redundant Chord } \\
\text { Lee, Jang, Lee } \\
\text { [44], } 2004 \\
\end{array}$ & Chord & AODV & Layered & $\begin{array}{l}\text { Random Way Point, } \\
\text { Speed: } 1 \mathrm{~m} / \mathrm{s} .\end{array}$ & $\begin{array}{l}\text { Compared in a simulation environment } \\
\text { with Chord. } 1000 \text { mobile nodes were } \\
\text { used with } 1 \mathrm{~m} \text { transmission range. }\end{array}$ \\
\hline $\begin{array}{c}\text { MRDP, } \\
\text { Liang, Chen, Zhang } \\
{[58], 2010}\end{array}$ & Clustering & AODV & Integrated & $\begin{array}{l}\text { Semi-Markov Smoth } \\
2-5 \mathrm{~m} / \mathrm{s} \text {. }\end{array}$ & $\begin{array}{l}\text { Compared to Ekta and VRR. } 50-200 \\
\text { nodes were simulated. }\end{array}$ \\
\hline $\begin{array}{c}\text { Bamboo/AODV, } \\
\text { Castro, Villanueva, } \\
\text { Ruiz, Sargento, } \\
\text { Andreas, Kass ler, } \\
\text { [59],2008 } \\
\end{array}$ & Bamboo & AODV & Layered & No & $\begin{array}{l}\text { Three configurations were evaluated; No } \\
\text { management, standard management and } \\
\text { custom management. Simulated } 36 \text { and } \\
49 \text { nodes. No comparison with other } \\
\text { systems }\end{array}$ \\
\hline $\begin{array}{l}\text { Convergence Chord } \\
\text { Mei, Ji, Li [60],2009 }\end{array}$ & Chord & AODV & Cross-layer & No & $\begin{array}{l}\text { Compared to conventional Chord over } \\
\text { MANET. } 30 \text { nodes were simulated. }\end{array}$ \\
\hline $\begin{array}{l}\text { Cramer, Fuhrmann, } \\
\text { Chord in mobile ad } \\
\text { hoc network, [53], } \\
2006\end{array}$ & Chord & DSR, and AODV & Layered & $\begin{array}{l}\text { Random Direction } \\
\text { Model; Speed: 0, 2, } \\
\text { and } 5 \mathrm{~m} / \mathrm{s}\end{array}$ & $\begin{array}{l}\text { For comparison, other flood-based } \\
\text { protocol was implemented and was also } \\
\text { compared to Chord over OLSR. }\end{array}$ \\
\hline $\begin{array}{l}\text { da Hora, Macedo, } \\
\text { Oliveira, Siqueira, } \\
\text { Loureiro, Nogueira, } \\
\text { Pujolle, [56],2009 }\end{array}$ & Chord & AODV & Layered & $\begin{array}{c}\text { Random Way Point } \\
0,0.25,0.5,1,2,4,8 \\
\mathrm{~m} / \mathrm{s}\end{array}$ & $\begin{array}{l}\text { Compared to Chord layered on AODV } \\
\text { without modification. } 50 \text { nodes were } \\
\text { used. }\end{array}$ \\
\hline
\end{tabular}

\subsubsection{GPS-based systems}

A number of P2P over MANET approaches consider nodes' locations using the Global Positioning System (GPS) and use this information in the routing algorithms. MPI (MultiLevel Peer Index) [61], MeshChord [62], Kummer et al. [63] and MANETChordGNP [64] are solutions that exploit Global Position System information to improve overlay operation in MANET. Some of them used MANET routing protocols that already use location information, whereas others use reactive routing protocols and add such functionality to the systems. The authors of MeshChord claim that using location information when assigning the logical ID of a peer contributes toward reducing Chord traffic. They further claim that most of Chord's traffic occurs between a peer and its predecessors/successors peers. Thus, assigning physical neighbour nodes with close-by logical ID will reduce the traffic in the network. In addition, the utilization of coordinate information is seen to help predicting network distance reducing routing stretch. However, employing a topology which is dependent on nodes' location causes issues when the nodes move. Node movement changes the neighbours of a node and hence require node ID and topology changes which in turn may require moving data between nodes as the changed IDs of nodes no longer match the data key. Furthermore, GPS is not available universally and scenarios where mobile nodes are inside buildings are difficult.

Multi-level Peer Index assumes that each mobile node knows its location through GPS. It divides the physical network into equal-sized logical grid cells with each cell being further divided to smaller equal-sized children cells. Data objects are hashed into geographical coordinates. For data lookups, a node sends a request to the index node in its level first. If not resolved (data outside current cell), the index node forwards the request to the index node in the next level up. The process continues until the highest level cell is reached or the index entry for the requested key is found. Once the index entry is found, a location lookup procedure is invoked at the cell where the index entry was found. The aim of the location lookup is to find the peer that is responsible for the peer ID of the source node. The reply to this request will contain the precise location of the node that stores the content. When a node moves from one grid cell to another, it deletes its old index information and obtains new index information from any node in the new grid cell. 
Table 3: GPS-based systems

\begin{tabular}{|c|c|c|c|c|c|}
\hline $\begin{array}{c}\text { System Name, } \\
\text { Authors, Reference }\end{array}$ & P2P system & MANET Routing & $\begin{array}{c}\text { Deployment } \\
\text { method }\end{array}$ & $\begin{array}{l}\text { Mobility Model and } \\
\text { Max Node Speed }\end{array}$ & Evaluation/Simulation \\
\hline $\begin{array}{c}\text { Multilevel Peer Index } \\
\text { Lee, Sivasubramaniam } \\
{[61], 2004}\end{array}$ & Grid structure & GPSR & cross-layer & $\begin{array}{c}\text { Random Way Point } \\
\text { Speed : ranging from } \\
0 \text { to } 20 \mathrm{~m} / \mathrm{s}\end{array}$ & $\begin{array}{l}\text { Simulated with csim simulator. Network } \\
\text { size: } 64 \text { - } 4096 \text { nodes with average } \\
\text { density of } 4 \text { nodes / } 175 \mathrm{~m} \times 175 \mathrm{~m} . \\
\text { Compared to flooding approach. }\end{array}$ \\
\hline $\begin{array}{l}\text { MESH CHORD } \\
\text { Burresi, Canali, } \\
\text { Renda, Santi, [62], } \\
2008 \\
\end{array}$ & Chord & DSR & Cross-layer & No & $\begin{array}{l}\text { Compared to Chord, Chord with location } \\
\text { awareness (ChordLoc) and Chord with } \\
\text { cross-layer design. 49-144 nodes were } \\
\text { simulated. }\end{array}$ \\
\hline $\begin{array}{l}\text { Kummer, Kropf, } \\
\text { Felber [63], } 2006\end{array}$ & $\begin{array}{l}\text { Logical ring } \\
\text { similar to Chord }\end{array}$ & $\begin{array}{r}\text { LAR (Location } \\
\text { Aided Routing) }\end{array}$ & - & No & $\begin{array}{l}\text { Static scenarios for networks of } 1000 \text {, } \\
10000 \text { and } 100000 \text { nodes. Evaluated } \\
\text { using LAR (Location-Aided Routing } \\
\text { protocol) with the positions of all nodes } \\
\text { being known through GPS. }\end{array}$ \\
\hline $\begin{array}{c}\text { MANETChordGNP } \\
\text { Fantar, Youss ef } \\
{[64], 2009}\end{array}$ & Chord & AODV & Integrated & Speed: 9 mps. & $\begin{array}{l}\text { Compared to Chord over AODV. } \\
\text { Network size: } 50 \text { - } 200 \text { nodes. }\end{array}$ \\
\hline
\end{tabular}

MeshChord has adopted Chord and uses non-location aided routing protocols at the network layer. However, location information was used in MeshChord to include awareness of physical proximity. MeshChord was proposed for mesh networks that have stationary nodes (upper tier architecture) and mobile clients (lower tier architecture). The upper tier architecture implements Chord to locate services within the network. Each stationary node takes on responsibility for a set of physically close mobile nodes. In order to exploit locality and assign close logical ID for peers who are physically close to each other, MeshChord uses location awareness by using GPS coordinates of the peers to calculate their logical peer IDs. Thus, two underlay neighbours are likely to also be close to each other in the overlay. However, Services are assigned an ID in as in traditional Chord. MeshChord adopts a cross-layering technique to speed up the process of key lookup. Each MeshChord peer is designed to pass up any received packet at the MAC layer even if it was not destined to this peer. The packet is processed by the application layer if it is a lookup request. Otherwise, it is discarded. In order to find a service, a mobile client sends the lookup request to its upper peer (the stationary node). Upon receiving the request, the stationary node uses Chord to find the key in the network. The reply is transmitted to the initiator of the request through the stationary node to which the initiator belongs.

The approach by Kummer et al. [63] constructs a DHT over a Location Aided Routing protocol with the position of all nodes being known. It builds a logical ring similar to Chord. However, the logical overlay is organised such that each peer maintains a minimalist logical overlay and ignores the logical long range neighbours to avoid high maintenance cost. For efficient lookups, it relies on physical neighbours to find long range neighbours. Routed lookups can be diverged from their original route if a shorter route is found by in intermediate peer by checking its logical and physical neighbours for a peer closer to the key. Peers receive information about their physical neighbours by exchanging neighbourhood information messages which are distributed using broadcasting.

MANETChordGNP also adopted Chord as the overlay. It uses a non-location aided routing protocol at the network layer. However, it uses location information to include awareness of physical proximity. MANETChordGNP considers physical locality through using the Global Network Positioning System (GNP). Chord is modified to position peers on the ring according to their GNP coordinate instead of the hash-based placement of Chord. In GNP, a set of hosts are chosen as landmarks to serve as references for other nodes who want to participate in the coordinate space. If one of the landmark nodes fails, the peer with the closest logical ID to the failing one takes over the role and acts as a landmark. Each peer is assigned a temporary unique overlay ID and whenever a peer changes its position, it requires a new overlay ID. MANETChordGNP maintains Chord routing tables and standard ADOV routing tables to carry out logical and physical routing. For the logical routing, a key lookup is destined to the closest logical peer in the overlay. In order to reach the next hop in the overlay, the underlay is consulted to carry out the physical routing. Table 3 present an overview of these systems.

\subsubsection{Underlay-Independent systems}

In this category, we introduce approaches which improve on the performance of overlay algorithms with respect to their use on mobile networks. As this covers a very broad range 
of approaches we limit our review to approaches which are applicable to MANET systems. M-CAN [65], M-Chord [66], Enhanced Mobile Chord (EMC) [67], Enhanced Backtracking Chord EBC [68], PNS-Chord [69] and Ring Interval Graph Search RIGS [70] do not work together with any specific MANET protocol, but aim at improving the performance of the overlay regardless of the underlying MANET protocol. These systems are presented in table 4.

M-CAN and M-Chord adopt the CAN and Chord P2P overlays respectively. Both systems use grouping and registration mechanisms to improve the performance. MCAN uses super-nodes to improve the performance of CAN in mobile networks. Super-nodes are selected nodes based on their capacity and connections reliability to manage a part of the ID space. Each group is restricted to manage a certain number of nodes. If the number of nodes in that group exceeds the maximum number, the group is divided. The super node for the new group is announced by the old super node. In order to join a M-CAN overly, a node registers itself with super nodes according to the IDs of the shared files. Thus, a node may register with more than one super node. To find a key, peers send their requests to their super-node which in turn forwards the request to the destination super node using the CAN algorithm. The destination super node checks its local directory to establish whether the destination node is registered with it. If the node is found, a reply with the destination address is sent to the request initiator. Otherwise, the request message gets transmitted to other groups by the destination.

M-Chord uses a hierarchical structure and registering mechanism to allow normal peers to register with superpeers which are selected depending on their computing capability and their connectivity. Ordinary nodes may register with more than one super peer. A peer that does not share files registers with a super node that has spare capacity. The logical space is distributed between super peers in typical Chord fashion and routing between these also uses standard Chord. In addition to maintaining a Chord routing table, each super peer also maintains a directory of shared files that were registered by ordinary nodes. Nodes send lookup requests to their super node, which routes the request using standard Chord to other super nodes. These super peers check their file directory for the requested key. If the requested key is found, a response to the initiator is generated which contains the address of the ordinary peer storing the file. Direct communication between the requesting peer and the node storing the file takes place to retrieve the file.

Enhanced Mobile Chord [67] modifies Chord to enhance its performance in MANET considering criteria such as delay, packet loss rate and jitter are taken into account. EMC includes a path selection mechanism based on a set of parameters such as application type and node stability. It uses periodic pinging to provide information about path latencies. Each EMC peer sends a periodic message to all successor peers in its finger table. Peers can compare latency etc to thresholds and select paths with appropriate latency values. Ping messages are also used to calculate a node stability value. For this, each peer keeps track of the number of received ping messages from a certain peer.

Similarly Enhanced Backtracking Chord builds a Chordbased overlay on MANET. It combines aspects of Enhanced Mobile Chord and Backtracking Chord. It uses the retransmission approach from Backtracking Chord which is claimed to enhance the success ratio of the P2P system. For each lookup request sent, the initiator sets a time-out timer. If this expires before a reply is received, a new copy of the request is sent. For the purpose of retransmitting the request, EBC peers check if there is a second best path, if so this is used for the retransmission. Otherwise, the strategy of backtracking Chord is used retransmitting the lookup request to the next successor in the finger table. Moreover, EBC also adopts the path selection mechanism from EMC. Again, periodic pinging is used to provide information about the latencies of routes. Chord's routing table is extended to store these additional routing parameters. For each entry, two extra fields are stored; node stability and path delay.

PNS-Chord has used Proximity Neighbour Selection to enhance the performance of overlay in ad hoc networks. PNS-Chord uses Proximity Neighbour Selection to mirror physical proximity in the logical Chord overlay. A node in PNS-Chord selects its logical neighbours considering their physical proximity which helps to avoid routing stretch. In PNS-Chord nodes maintain information about their physical neighbouring nodes. For this nodes exchange hello-like messages at the underlay routing level. Each node broadcasts a periodic hello message to its neighbours. These hello messages contain information about the nodes known neighbours. This provides nodes with information on neighbouring nodes up to 2 hops away. Such information is then provided to the overlay to build a logical structure that better matches the physical structure.

Ring Interval Graph was used by RIGS. The approach presented in [70] builds an underlay-dependent DHT where one hop neighbours in the overlay are also one hop neighbours in the underlay topology. Node IDs are not assigned in a random way; but rather in a way that generates a Ring Interval Graph RIG. For this a spanning tree of the entire network is needed. Upon constructing the spanning tree, each node is assigned an ID in an increasing order along the spanning tree. Once the Ids are assigned, a circular logical space as in Chord is built for the overlay system. A key item is hashed and assigned to the peer with equal or greater logical ID in the logical space. RIGS uses a continuous interval hash space $[0,1)$ instead of the discrete set used by Chord $\left\{0,1, \ldots 2^{m}\right\}$. When a node joins or leaves the system, the RIG and the overlay needs to be reconstructed so the matching between the overlay and underlay is maintained. 
Table 4: Underlay-Independent systems

\begin{tabular}{|c|c|c|}
\hline $\begin{array}{c}\text { System Name, Authors, } \\
\text { Reference }\end{array}$ & P2P system & Evaluation/Simulation \\
\hline $\begin{array}{c}\text { M-CAN } \\
\text { Peng, Li, Jin, Ma, } \\
{[65], 2004} \\
\end{array}$ & CAN & $\begin{array}{c}\text { Compared to flooded requests protocol and } \\
\text { centralized directory protocol. 50-500 peers were } \\
\text { used in the simulation. }\end{array}$ \\
\hline $\begin{array}{l}\text { M-Chord } \\
\text { Li, Chen, Sheu } \\
{[66], 2006} \\
\end{array}$ & Chord & $\begin{array}{l}\text { Comparison was done between normal Chord, } \\
\text { super M-Chord nodes and ordinary M-Chord } \\
\text { nodes. No. of nodes varies from } 128 \text { to } 2048 \text {. }\end{array}$ \\
\hline $\begin{array}{c}\text { Enhanced Mobile Chord } \\
\text { Thaalbi, Bejaoui, Tabbane, } \\
{[67], 2011}\end{array}$ & Chord & $\begin{array}{l}\text { Compared against native Chord and } \\
\text { Backtracking Chord. Simulates } 100 \text { nodes in a } \\
\text { dynamic network" by changing the churn rate" } \\
\text { and } 50-250 \text { nodes in a static network. }\end{array}$ \\
\hline $\begin{array}{l}\text { Enhanced Backtracking } \\
\text { Chord } \\
\text { Thaalbi, Tabbane, Bejaoui, } \\
\text { Meddahi, [68],2012 }\end{array}$ & Chord & $\begin{array}{l}\text { Compared to EMC and Backtracking Chord in } \\
\text { static network consists of } 50-250 \text { nodes and in a } \\
\text { dynamic network" by changing the churn rate } \\
\text { "that consists of } 100 \text { nodes. }\end{array}$ \\
\hline $\begin{array}{c}\text { PNS-Chord } \\
\text { Cramer and Fuhrmann } \\
\text { [69],2005 }\end{array}$ & Chord & $\begin{array}{l}\text { Simulated but not compared to other systems. } \\
\text { 100-1000 nodes were used but no mobility } \\
\text { model nor speed was simulated. }\end{array}$ \\
\hline $\begin{array}{c}\text { Ring Interval Graph Search } \\
\text { Shin and Arbaugh } \\
{[70], 2009}\end{array}$ & Chord & $\begin{array}{l}\text { Compared to Chord, VALLEYW ALK } \\
\text { (unstructured P2P for ad hoc) } .100 \text { nodes were } \\
\text { simulated in a stitic network. }\end{array}$ \\
\hline
\end{tabular}

\subsection{P2P-Based MANET routing protocol systems}

This group of approaches use techniques from P2P overlays to improve MANET underlay routing protocols. These approaches do not use the DHT in the application layer, but at the network layer to enhance the scalability of MANET routing. In such setting, the DHT is used to distribute MANET nodes location information throughout the network. This information would reflect the topological position of the nodes. However, some of these systems support traditional $\mathrm{P} 2 \mathrm{P}$ functionality like resources distribution and discovery but at the network layer. Approaches falling into this category can be further divided according to the P2P structure used. There are Ring-Based systems, Tree-Based systems and systems based on other structures. Virtual Ring Routing VRR [71], Scalable Source Routing SSR [72], DPSR [73], Ekta [46], MADPastry [74] and MA-Chord [75] belong to the Ring-Based MANET routing systems (Table 5). Tribe [76], DART [78], Automatic Incremental Routing ATR [79], Indirect Treebased Routing ITR [80], and KDSR [82] are Tree-Based MANET routing approaches (Table 6). DHT-based functionality using Hypercube [77], AIR [81] and 3D-RP [83] are approaches which adopt various structures such as hypercube, acyclic graph and 3D rectangular structure (Table 7).

\subsubsection{P2P Ring-Based MANET routing systems}

VRR [71] uses location independent IDs. It organizes nodes into virtual ring similarly to Chord and Pastry. VRR supports traditional point-to-point routing and DHT routing. VRR nodes maintain paths to their virtual neighbours and set of physical neighbours. A VRR node maintains a virtual neighbour set which is divided into two halves. The first half stores its closest neighbours clockwise the logical space. The second half is used to store its closest nodes anticlockwise around the identifier space. Each routing entry in the virtual neighbour set records the address of the next physical node and the address of the virtual neighbour. Nodes also maintain a physical neighbour set to store nodes which are in the communication range of this node. Routing tables are updated in a proactive way.

Similarly, SSR [72] creates a Chord-like ring at the network layer. SSR nodes maintain their physical neighbours, virtual successors, virtual predecessors, and some cached information. SSR employs indirect routing using DHT-like routing and direct routing between nodes. To route a packet to a destination, a node consults its routing tables to find the destination or a node closer to the destination. The route cache stores source routes to the virtual successors and predecessors. Each transmitted data packet follows a source route. The route does not necessary lead to the final destination but to a virtual node which is closer to it. Such source routes can be altered by intermediate virtual nodes if a shorter route is available. According to the presented 
results, this approach suits growing networks that are supported by some fixed wired nodes. It is most effective at low mobility.

DPSR [73] proposes a MANET source routing protocol integrating Pastry with DSR. It limits the number of source routes that a node needs to maintain to $\mathrm{O}(\log \mathrm{N})$. Each node is assigned an ID by hashing its address. It changes the content of the routing table entry and leaf set of the original Pastry algorithm to store the source route to reach a destination instead of storing its IP address as in Pastry. DPSR routes a lookup request similarly to Pastry where a key is routed to the node with the closest logical ID. It also adopts the same routing table maintenance mechanism as Pastry where for each row in the routing table DPSR picks a random entry from the row and ask that node for a copy of the corresponding routing table entry.

Ekta [46] and MADPastry [74] have also integrated Pastry with DSR and AODV routing protocols, respectively, at the network layer. Ekta is an enhancement of DPSR. It builds a separate overlay at the network layer and does not use the DHT for unicast routing. Similarly to DPSR, Ekta modifies Pastry routing tables to store source routes for each entry instead of the destination's IP address. Prefix based routing is used in Ekta to route a key to a node with logical ID closer to the destination. Ekta inherits the functionality of DSR for route discovery and maintenance. For updating routing table entries, Ekta optimises overhead and forwarded packets to maintain fresh routes. MADPastry combines AODV and Pastry at the underlay to provide indirect routing for MANET. It utilizes Random landmarking to group physical neighbour nodes in a cluster.
A set of nodes in the same physical cluster share a common overlay ID prefix. MADPastry divides the space into landmark keys. A node can be temporarily the landmark and takes on responsibility for a set of landmark keys. In case of landmark node failure, a node in the same cluster with an ID closest to the failing node will take the responsibility for the landmark keys. Landmark nodes broadcast a periodic message to allow other nodes to measure their distance from it. In order to limit the traffic, such messages are forwarded only by nodes that belong to the cluster. Based on the periodic messages, nodes can establish which landmark they are closest to and possibly reregister with a new landmark node. MADPastry maintains three routing tables; AODV routing table, Pastry routing table and leaf set for indirect routing.

MA-Chord [75] combines AODV and Chord at the network layer to provide DHT routing in MANET. It applies random landmarking and uses fixed landmark keys to exploit physical proximity. MA-Chord maintains AODV routing tables to serve physical routing. In addition, it also manages Chord routing tables. The Chord routing table is maintained in a proactive way where each MA-Chord node sends periodic messages to its successors and predecessors in the overlay. Routing entries are also updated through overhearing network traffic. Each node is assigned an overlay ID when it joins the overlay. Each node publishes its own overly ID with an overlay key node which works as a temporary address server. For routing purposes, a node needs first to retrieve the destination's overlay ID from the destination's address server. After retrieving the overlay ID, a node can then use it with MA-Chord overlay routing.

Table 5: P2P Ring-Based MANET routing protocol systems

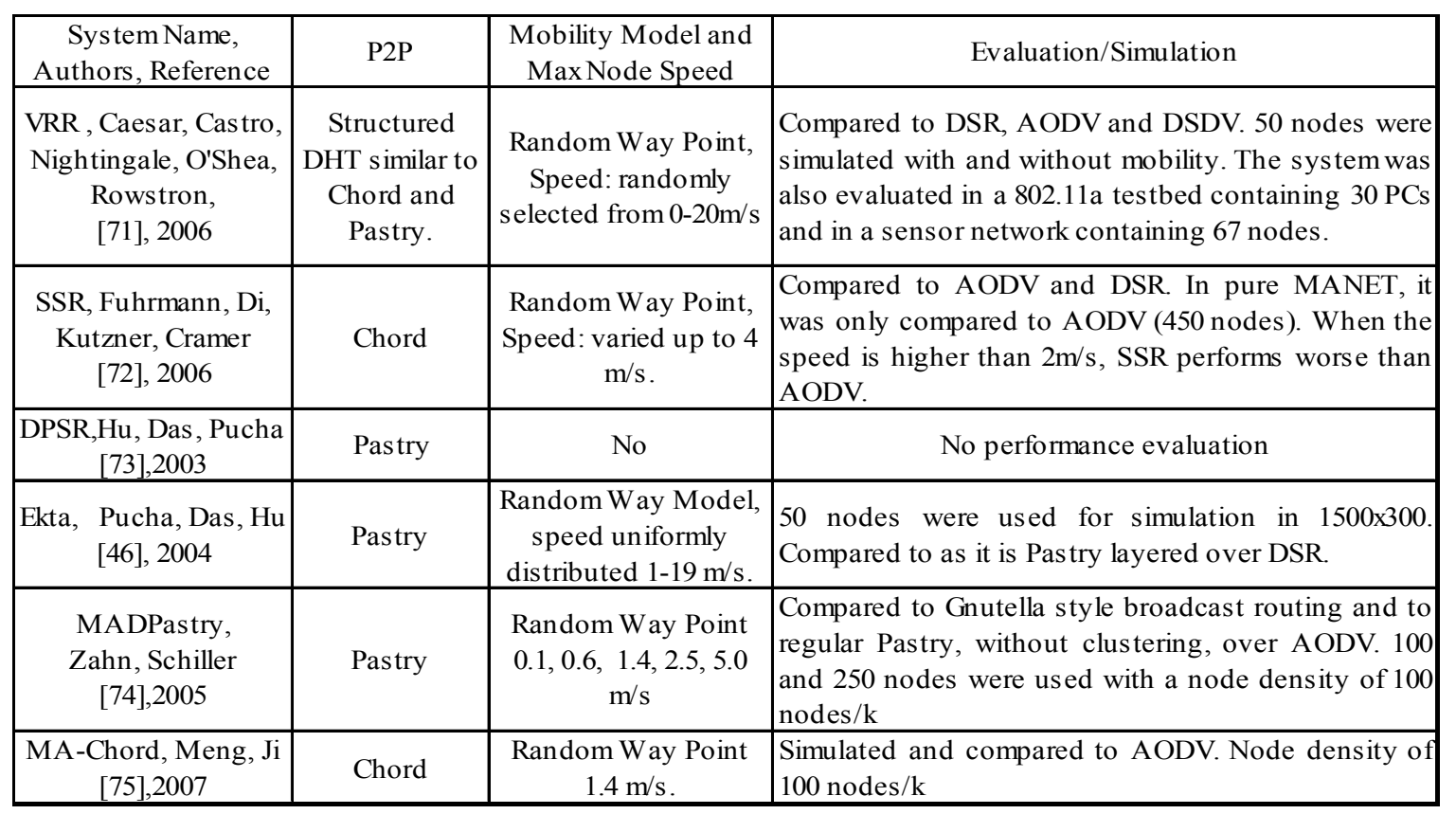




\subsubsection{P2P Tree-Based MANET routing systems}

Tribe [76] builds a tree-like logical structure of the physical topology. Neighbouring nodes in the underlay topology are represented in a close logical region of the address space. Each Tribe node maintains information about itself, its immediate neighbours and any nodes under its responsibility. Each tribe node has three identifiers; universal ID, logical Tribe ID and relative ID. The relative address is topology dependant and changes when nodes move to another location in the network. When a node joins the network, one of its physical neighbours, with the largest portion of the ID space, will share its logical space with the new node. The joining node then needs to register with an anchor node which is responsible for its logical ID. The anchor node stores information about the joining node such as its location (relative ID). To find a node, a source node contacts the destination's anchor node to get the location of the destination. It finds the responsible anchor node for a destination by hashing the destination's universal ID.

DART [78] builds a logical tree structure on top of an underlay to implement hierarchical routing. The constructed logical tree does not represent the exact physical links between nodes. However, neighbouring nodes in the logical address space should be relatively close to each other in the physical topology. Each DART node has its routing address and a permanent identifier. Routing addresses may change due to node movement as they are assigned according to node position. Any branches of a leaf share a prefix of the routing address with its vertex leaf. It maintains a routing table that stores an entry for each sibling using proactive distance-vector maintenance. A DART node needs to register itself (identifier, routing address) with another node that acts as an anchor node. To achieve this, a node hashes its identifier and the result is the address of the anchor node where this node should store its (identifier, routing address) pair. For routing, a node can hash the destination's identifier to find its anchor node where the destination's routing address is stored.

ATR [79] builds a similar structure to DART where it builds a binary tree of $L+1$ levels ( $L$ being the number of address bits). In the tree structure, the set of nodes in the same sub tree level share an address prefix. Thus the number of shared bits in nodes' addresses expresses the distance between them in the topology. ATR augments the tree structure to offer more than a single path between nodes. ATR maintains and explores all the possible paths through its neighbours. It uses periodic hello messages to build and update the routing table. An ATR routing table stores multiple entries per sibling to achieve multi path routing in the tree structure.
Similarly to DART, a DHT is used for address lookup. Each ATR node registers its network address and identifier with a node in the network depending on the hash value of its identifier. When a node wants to retrieve the network address of a destination, it hashes the identifier of the destination to find the node which is responsible for this destination. It can then get the destination's network address from that node.

Indirect Tree-based routing ITR [80] extends Augmented Tree-based Routing ATR [79]. ITR presents the identifier space as a tree structure to assure agreement between logical and physical structure. It is quite similar to its predecessor. However, it provides full functional P2P services where nodes can store resources and fetch them. It assigns each ITR node a location-dependant identifier. It divides the routing table into as many sections as the number of the siblings. An n-th section stores the next physical hop which can be used to deliver a packet to the level $n$ sibling in the logical space. Nodes need to register their identifier with an anchor node periodically as in ATR. The same procedure applies for shared services with a node being required to periodically send a pair of (resource identifier, storing peer identifier) to the node responsible for this service.

KDSR [82] integrates the functionality of Kademlia with DSR to provide indirect and direct routing in MANET. KDSR adopts Kademlia XOR-based algorithm. It also builds a route cache and adopts the use of non-propagating route requests to minimize network traffic. Each KDSR node maintains a k-bucket routing table as in Kademlia. However, the content of each entry is modified to suit MANET. Each entry stores a four tuple that consists of node ID, IP address, XOR distance to the node and the source route to the node. In addition, KDSR also maintains a cache route similar to DSR. KDSR optimizes k-bucket update mechanism to learn new source routes which will be stored in the cache.

\subsubsection{MANET routing systems employing other P2P structures}

Alvarez [77] constructs a hypercube-like address space to build a multi path unicast routing. It translates the physical network into a logical network through the use of node coordinates in a hypercube. Each node in the hypercube manages a portion of the address space which can be split with any joining node. For each dimension, a node in the hypercube must have a link to another node. For example a node in the hypercube with logical $(0,0,0)$ coordinate has links to nodes with coordinate $(0,0,1),(0,1,0)$ and $(1,0,0)$. These nodes differ from $(0,0,0)$ in 1 dimension only. 
Table 6: P2P Tree-Based MANET routing protocol systems

\begin{tabular}{|c|c|c|c|}
\hline $\begin{array}{l}\text { System Name, } \\
\text { Authors, Reference }\end{array}$ & $\mathrm{P} 2 \mathrm{P}$ & $\begin{array}{l}\text { Mobility Model and } \\
\text { Max Node Speed }\end{array}$ & Evaluation/Simulation \\
\hline $\begin{array}{l}\text { Tribe, Viana, Amorim, } \\
\text { Fdida, [76],2004 }\end{array}$ & Tree structure & - & $\begin{array}{l}\text { evalauted in simulation but was not compared to any } \\
\text { other system. Simulate a } 1000-10000 \text { network size. }\end{array}$ \\
\hline $\begin{array}{l}\text { DART, Eriks son, } \\
\text { Faloutsos, } \\
\text { Krishnamurthy, [78], } \\
2007\end{array}$ & Tree structure & $\begin{array}{l}\text { Random Way point, } \\
\text { random speed } \\
\text { between } 0.5 \mathrm{~m} / \mathrm{s} \text { and } 5 \\
\mathrm{~m} / \mathrm{s}\end{array}$ & $\begin{array}{l}\text { compared to AODV, DSR and DSDV with up to } 800 \\
\text { nodes. }\end{array}$ \\
\hline $\begin{array}{l}\text { ATR, Caleffi, } \\
\text { Ferraiuolo, Paura, } \\
\text { [79], } 2007\end{array}$ & Tree structure & $\begin{array}{l}\text { Random Way point, } \\
\text { random speed } \\
\text { between } 0.5 \mathrm{~m} / \mathrm{s} \text { and } 5 \\
\mathrm{~m} / \mathrm{s}\end{array}$ & compared to DART, AODV and DSR in simulation \\
\hline $\begin{array}{l}\text { KDSR, Zhao, Wen, } \\
\text { Zhao, [82], } 2009\end{array}$ & Kademlia & $\begin{array}{l}\text { Random way point, } \\
\text { random speed } \\
\text { between } 0 \text { and } 20 \mathrm{~m} / \mathrm{s} \text {. }\end{array}$ & $\begin{array}{l}\text { compared to DSR in a simulation with } 50 \text { nodes } \\
\text { network size. }\end{array}$ \\
\hline
\end{tabular}

AIR [81] builds a Labelled Directed Acyclic Graph (LDAG). The LDAG structure is constructed with reference to an elected root node. AIR maintains a DHT to minimize the use of flooding in MANET. The DHT is used to provide mapping of a node identifier such as an IP address to a prefix label. It adopts prefix based routing where a node fetches the prefix label of a destination node. Each AIR node hashes its identifier to get its prefix label and then announce its presence by sending publish message to its anchor node which is the node that best matches its prefix label. Each node in the network maintains a neighbour table, two-hop neighbour table and a multicast group table. AIR uses hello messages as control messages to exchange information between nodes.
3D-RP [83] builds a three-dimensional logical structure on top of the network topology. A 3D-RP node calculates its mbit logical ID in form of a tuple $\{x|y| z\}$. Hello messages are exchanged between one hop physical neighbours to maintain the 3D logical space. These Hello messages contain information about the node including logical ID, universal ID (IP address), logical space portion and its logical one hop neighbours. Using a hash function, a node can identify its anchor node by hashing its universal ID (IP). Once the anchor node was identified, the node can store its logical identifier and its universal identifier on the anchor node. Thus, allowing other nodes in the network to find it using its logical identifier. 3D-RP nodes route messages towards their logical neighbour with the closest position in every tuple $\{x|y| z\}$.

Table 7: MANET routing protocol systems based on other P2P structures

\begin{tabular}{|c|c|c|c|}
\hline $\begin{array}{l}\text { System Name, } \\
\text { Authors, Reference }\end{array}$ & $\mathrm{P} 2 \mathrm{P}$ & $\begin{array}{l}\text { Mobility Model and } \\
\text { Max Node Speed }\end{array}$ & Evaluation/Simulation \\
\hline $\begin{array}{c}\text { DHT-based } \\
\text { Functionalities Using } \\
\text { Hypercubes, Alvarez- } \\
\text { Hamelin, Viana, } \\
\text { Amorim, [77], } 2006\end{array}$ & $\begin{array}{l}\text { Hypercubes } \\
\text { structure }\end{array}$ & - & - \\
\hline $\begin{array}{c}\text { AIR, Garcia-Luna- } \\
\text { Aceves, Sampath, } \\
{[81], 2009} \\
\end{array}$ & $\begin{array}{c}\text { Labeled } \\
\text { directed } \\
\text { acyclic graph }\end{array}$ & Random way point & compared to AODV and OLSR as unicast routing. \\
\hline $\begin{array}{l}\text { 3D-RP, Abid, } \\
\text { Othman, Shah, [83], } \\
2013\end{array}$ & $\begin{array}{l}\text { 3D rectangular } \\
\text { structure }\end{array}$ & \begin{tabular}{|} 
random way point, \\
Random speed \\
between $0.4 \mathrm{~m} / \mathrm{s}$ and 1 \\
$\mathrm{~m} / \mathrm{s}$.
\end{tabular} & $\begin{array}{l}\text { compared to M-DART in a simulation with } 25-400 \\
\text { nodes. }\end{array}$ \\
\hline
\end{tabular}




\section{Discussion}

\subsection{Adopted P2P system}

Considering the used overlay algorithms, we find that most of them are well known algorithms commonly used on wired networks. They were introduced in MANET context to support either data dissemination or location distribution for MANET routing purposes. However, most of the proposed system concluded that straight deployment of wired internet technology for P2P does not suit MANET networks. Therefore, most of the proposed systems have introduced modifications to the original P2P technology to suit MANET. Chord, Pastry, CAN, Bamboo and Kademlia were used and modified to work in MANET. Specifically, Chord and Pastry were the most commonly used DHT protocols on MANET for data distribution in mobile ad hoc networks. About $50 \%$ of the studied systems have used Chord or a system that builds a logical structure similar to Chord. Pastry was also used by well-known systems such as CrossROAD, DPSR, Ekta and MADPastry. Moreover, the tree structure of the logical overlay was also adopted by number of proposed systems. RIGS, MPI, DART, ATR and ITR are examples of systems that build an overlay with a tree structure. They build such a structure at the network layer to support scalable MANET routing. However, such a tree structure typically creates substantial traffic, especially when considering node mobility as this frequently requires reconstructing the overlay. Another issue with tree structures is the possibility of a disconnected sub set of the network in the event of node failure. This is due to single paths between nodes in the tree structure. To overcome this weakness, ATR modified tree structure to support multiple paths between nodes in the network. In addition to the aforementioned logical structures, some of the studied systems built 3D- rectangular and hypercube structures.

\subsection{Clustering and registration}

A number of systems make use of a clustering mechanism to structure the overlay. Examples of systems that follow this paradigm are M-Chord, M-CAN, MADPastry, MRDP, P2P MMOG, ROUBST and MA-Chord. Some of these systems employ a second mechanism to organise the cluster heads. For instance, M-Chord uses Chord among super peers and M-CAN adopts CAN to organise cluster heads.

A drawback of using clustering is that it requires the cluster members to register with the cluster head. The registration can be done the first time a peer publishes a file as in MCAN and M-Chord. Each super-peer is allocated a range of IDs it is responsible for. When publishing a file, a peer calculates the ID of the file then registers with the superpeer who is responsible for this file's ID. This approach is independent of a node's physical location. Another form of registration requires peers to register each time a peer changes location and moves into the territory of a different cluster. Clearly, in networks with substantial node mobility such an approach creates significant overhead and would not be efficient. However, it may be advantageous in mostly static networks.

\subsection{Consideration of physical proximity}

Generally, constructing a P2P over MANET system which operates independent of the physical proximity of nodes leads to a longer path of messages at the MANET layer. This is due to the fact the nodes which are close in the constructed virtual network may be physically distant. So a hop in the overlay translates to a path in the underlay. Thus, building a P2P over MANET system considering the physical proximity between nodes, i.e. the overlay closely maps the physical topology, can substantially reduce the traffic in the network. Examples of approaches that consider nodes proximity are MADPastry, Hashline, PNS-Chord, RIGS, MRDP, P2P MMOG, MA-Chord and MANETChordGNP.

Mapping the physical topology closely at the overlay can result in more traffic, especially when node mobility is high. In order to maintain the relationship between the overlay and the underlay, additional maintenance traffic is required. In order to consider physical proximity, some of the proposed systems use landmarking, clustering or building logical tree structures. However, with the presence of node mobility, it is more expensive to maintain the consistency of the network. This problem is exasperated in systems that use logical IDs which are based on nodes' location.

On the other hand, some of the systems do not consider physical node proximity. They build overlay networks independent of the MANET physical topology. These approaches introduced some modifications to existing $\mathrm{P} 2 \mathrm{P}$ algorithms to adapt to MANET networks. EMC, Enhanced Backtracking Chord, Backtracking Chord, Redundant Chord, M-CAN and CrossROAD are examples of such systems.

\subsection{Deployment strategy}

The first and easiest way is to deploy the overlay directly on top of the existing MANET architecture without any direct communication between the layers. This design conforms to the OSI layering architecture. The layered approach was used by Backtracking Chord, Redundant Chord and Bamboo/OADV. Backtracking Chord and Redundant Chord introduce some modifications to $\mathrm{P} 2 \mathrm{P}$ Chord protocol to improve the efficiency of the proposed system. However, systems that follow the layering approach run logical routing fully independent of the physical underlay routing. This type of deployment usually results in nodes that are logically close in the overlay to be physically distant in the underlay. This substantially increases lookup traffic and hence results in a comparably poor performance of the system.

Another approach is the integration of the P2P protocol with the underlay routing protocol. Examples of systems that 
have adopted the integration approach are DPSR, Ekta which was an enhancement of DPSR, MADPastry, Hashline, MRDP, VRR, SRR, Tribe, DART, ATR, ITR, 3D-RP and MA-Chord. Systems that follow this type of deployment have different purposes. Some of them were proposed to provide $\mathrm{P} 2 \mathrm{P}$ functionality at the MANET routing layer. However, most of the systems that employed DHTs at the network layer aimed at improving the routing performance. Examples of systems are VR, Tribe and ITR. However, it has been argued that since P2P overlays and MANET protocols have a number of commonalities, common information should be exploited to improve the performance of combined systems. This resulted in the cross-layering where P2P overlays operate at the application layer and MANET protocols operate at the network layer, with the two systems sharing information. To enable the exchange of such information a violation of the OSI architecture is needed. By permitting nonadjacent layers to communicate significant performance improvements can be achieved due to the reduction of duplicate efforts in the two layers. For example, CrossROAD uses an external data sharing module which holds the shared information. Another example is MA-SP2P where underlay routing information were used to identify number of physical hops between logical peers.

\subsection{Network routing protocol}

In regard to the adopted underlay routing protocols, they generally belong to three MANET routing protocol categories, which are proactive, reactive, and locationbased routing protocols. However, some of the studied systems have proposed their systems as MANET underlay routing protocol.

The most popular category in the studied systems is the reactive routing protocols which establish routes on demand. Especially AODV and DSR seem to be very popular. They were used by P2P MANET systems that adopted the layering and cross-layering strategy. In addition, they were also integrated with DHT at the network layer in some system to enhance MANET routing and provide direct and indirect routing in MANET. AODV was used by MADPastry, Backtracking Chord, Redundant Chord, MRDP, MA-Chord and MANETChordGNP. DSR was used by DPSR, Ekta, MeshChord, Hashline, KDSR and SSR. On the other hand, OLSR as a proactive protocol was used by some of the analysed systems. Despite the fact that there are a number of proactive protocols, OLSR is the only used protocol for deploying P2P over MANET across the studied system. Systems which employ a proactive routing protocol (OLSR) are CrossROAD, MA-SP2P, 3D and ROBUST. Compared to proactive protocols, reactive systems result in a higher routing latency since they establish routes to other nodes on demand. However, perhaps rather unexpectedly, reactive systems can also result in a higher load on system than proactive systems. This is especially true in more dynamic networks with high demand where many route requests and replies propagate throughout the network [53]. Moreover, some of the reviewed systems have used routing protocols that optimize node location information through the use of global positioning system GPS (LAR and GPSR). The use of GPS information would help to predict network distance when routing hence reducing routing stretch. However, building a logical ID that depends on the global position of a node requires increased overheads to maintain the consistency of the network when nodes move requiring a new logical ID and reassigning contents to different nodes.

\subsection{Performance evaluation}

Most of the studied systems have used network simulation tools for evaluation. In order to evaluate their proposed systems they usually simulate the system, and then compare it to other systems. The compared to system is sometime another $\mathrm{P} 2 \mathrm{P}$ over MANET solution, MANET routing protocol or straight layering of normal P2P protocol over MANET. Another approach for evaluation that was adopted is to compare the efficiency of the proposed system to mobile ad hoc routing protocol. This was quite popular for systems that introduced their solutions as replacement of traditional MANET routing.

However, not all the studied system has used the simulation tools to evaluate the proposed system. In fact some of them have used testbed containing a small number of nodes for the evaluation purposes instead of using simulation environment. For example, CrossROAD used eight nodes in a testbed experiment to analyse the performance of the system. Another example that was evaluated using testbed is VRR where 30 PCs were used in the performance evaluation. In addition, some of the proposed systems did not evaluate the performance of their solutions. For example, DPSR and P2P MMOG over MANET did not evaluate their systems.

A number of proposed systems have not included node mobility in their evaluation. For example, [63] and PNSChord did not consider mobility. Clearly, simulating static networks does not reflect the performance of the system in MANET with node mobility. However, some of the proposed systems have studied the effect of churn where nodes join and leave a static network and consider it as mobility in the network. Other systems consider mobility and they state how much velocity was considered when simulating their systems. Looking at the considered mobility we can find that most of the systems, who considered mobility, consider a node speed that is randomly selected from the range $0 \mathrm{~m} / \mathrm{s}-2 \mathrm{~m} / \mathrm{s}$. Few of the studied systems have considered a higher mobility up to $20 \mathrm{~m} / \mathrm{s}$. Ekta and MPI choose the mobility speed randomly up to about 20 $\mathrm{m} / \mathrm{s}$.

It appears that most of the simulation-evaluated systems use the random way point mobility model to simulate mobile nodes movement within the simulated area. 


\subsection{Future Research Directions}

Adaptation of P2P technology to ad hoc networks has been investigated for a number of years. P2P systems were used together with MANET for mainly two reasons. Firstly, to enhance MANET routing efficiency through the use of distributed hash tables. With DHT and location information being available at the routing layer, more scalable MANET can be supported. Secondly, P2P technology has been deployed on MANET to improve the support for data storage and retrieval for applications such as file sharing (Audio, video and news). Here overlays are placed at the application layer. Current systems aim at avoiding mismatches between the overlay and underlay through constructing the overlay reflecting the physical topology. Thus, physical neighbours are logically close as well. For largely static MANET with limited node mobility, this reduces the required maintenance traffic and overhead introduced through the use of DHTs. However, for dynamic overlays with a significant level of node mobility, this may actually result in an increased level of maintenance traffic as the overlay needs reorganisation in line with the physical topology. Consequently, the issue of MANET node mobility in P2P/MANET networks requires further investigation.

Despite the fact that some systems adopt cross-layering, the resulting synergies between the two technologies need to be exploited more. Specifically, exploitation of the underlay routing protocol information when building the overlay need to be enhanced. This would contribute towards building a less costly overlay.

Results reported in research papers are typically based on experimentation or simulation of rather small networks. Some setups employ only a single digit number of nodes. Indeed tool support for larger simulations is lacking. Key simulation tools struggle with the complexities of MANET and $\mathrm{P} 2 \mathrm{P}$ routing and larger networks require a huge amount of simulation system resources, which are impractical at best or, more often, impossible to meet. Simulation tools which support larger network sizes are needed to further research into P2P/MANET systems.

So far work has concentrated on using multi-hop overlays with MANET. Most MANET networks are characterised by a relatively small number of participating nodes which may be mobile. Consequently, investigating combining MANET with a one hop overlay might be worthwhile. Considering small to medium sized MANET networks (up to 200 nodes), such a solution is not prohibitively expensive, especially, when used on a proactive underlay [84]. The big advantage of such systems is the reduced latency for lookup operations.

Despite the fact that there are a good number of proposed P2P systems for mobile ad hoc, there has been only a limited focus on security in such systems. Clearly, security is a challenging topic in distributed systems such as P2P. However, it is even more challenging when $\mathrm{P} 2 \mathrm{P}$ are combined with MANET due to dynamicity and the use of wireless communication. Security in P2P over MANET requires more consideration.

Finally, cellular data networks together with increased power of mobile phones offer another research opportunity. Especially, initiatives such as the $5 \mathrm{G}$ project will increase the potential to integrated cellular networks with mobile ad hoc networks. This could extend substantially the reach of file and data sharing applications and requires further consideration.

\section{Conclusion}

This paper has investigated the current trend of deploying structured P2P over mobile ad hoc network. Overall, there are a good number of proposed solutions that looked at the adaptation of existing P2P technology into MANET. We classify the reviewed systems into two categories according to the use of DHT; DHT to enhance MANET routing and DHT to build P2P overlay over MANET. Systems that fall under the latter were further grouped with regard to the underlying MANET systems. Systems which were based on reactive underlay, proactive underlay, GPS-based systems and systems that do not specify their underlay. Generally, most of them utilized the most popular structured P2P for the wired internet; Chord, Pastry, Kademlia, Bamboo and CAN. Clearly, systems have been developed for different scenarios (network size, mobility, number of lookups etc). Yet there is little work investigating the use of one hop or variable hop overlay algorithms in this context. Furthermore, most system evaluations restrict network sizes to hundreds nodes. Unfortunately, the particular strength of $\mathrm{P} 2 \mathrm{P}$ overlays to scale to large networks is not mirrored in many experiments. Thus more work is required testing systems with larger networks.

\section{REFERENCES}

[1] C. Gottron, A. König, and R. Steinmetz, "A Survey on Security in Mobile Peer-to-Peer Architectures-Overlay-Based vs. Underlay-Based Approaches," Futur. Internet, vol. 2, no. 4, pp. 505-532, 2010.

[2] S. A. Abid, M. Othman, and N. Shah, "A Survey on DHT-Based Routing for Large-Scale Mobile," ACM Comput. Surv., vol. 47, no. 2, pp. 1-46, 2014.

[3] G. Fersi, W. Louati, and M. Ben Jemaa, "Distributed Hash tablebased routing and data management in wireless sensor networks: a survey,” Wirel. Networks, Vol. 19 (2), pp. 219-236, Jun. 2012.

[4] L. Liu, Y. Jing, Y. Zhang, and B. Xia, "A Survey on P2P File Sharing Algorithms over MANETs," Consum. Electron. Times, vol. 2, pp. 109-115, 2013.

[5] J. Van Der Merwe, D. Dawoud, and S. McDonald, "A survey on peer-to-peer key management for mobile ad hoc networks," $A C M$ Comput. Surv., vol. 39, no. 1, pp. 1-45, 2007.

[6] M. C. Castro, A. J. Kassler, C.-F. Chiasserini, C. Casetti, and I. Korpeoglu, "Peer-to-Peer Overlay in Mobile Ad-hoc Networks," in Handbook of Peer-to-Peer Networking, X. Shen, H. Yu, J. Buford, and M. Akon, Eds. Boston, MA: Springer US, 2010.

[7] M. Gerla, C. Lindemann, and A. Rowstron, "P2P MANET's-New Research Issues.," in Peer-to-Peer Mobile Ad Hoc NetworksDagstuhl Seminar, 2005, pp. 1-25. 
[8] Q. H. Vu, M. Lupu, and B. C. Ooi, Peer-to-peer computing. Principles and applications. Springer 2010.

[9] J. F. Buford and H. Yu, Handbook of Peer-to-Peer Networking. Springer US, 2010.

[10] J. F. Buford, H. Yu, and E. K. Lua, P2P Networking and Applications. Morgan Kaufmann, Elsevier, 2009.

[11] D. Korzun and A. Gurtov, Structured Peer-to-Peer Systems : Fundamentals of Hierarchical Organization, Routing, Scaling, and Security. New York, NY: Springer New York, 2013.

[12] K. Dhara, Y. Guo, M. Kolberg, and X. Wu, "Overview of Structured Peer-to-Peer Overlay Algorithms," in Handbook of Peer-to-Peer Networking, Springer US, 2010, pp. 223-256.

[13] S. Ratnasamy, P. Francis, and M. Handley, "A scalable contentaddressable network," in SIGCOMM, 2001, pp. 161-172.

[14] I. Stoica, R. Morris, D. Karger, M. F. Kaashoek, and H. B. Ý, "Chord : A Scalable Peer-to-peer Lookup Service for Internet," in SIGCOMM, 2001, pp. 149-160.

[15] A. Rowstron and P. Druschel, "Pastry: Scalable, decentralized object location, and routing for large-scale peer-to-peer systems," in Middleware, vol. 2218, Springer, 2001, pp. 329-350.

[16] H. Shen, C. Z. Xu, and G. Chen, "Cycloid: A constant-degree and lookup-efficient P2P overlay network," Perform. Eval., vol. 63, no. 3, pp. 195-216, 2006.

[17] A. Gupta, B. Liskov, and R. Rodrigues, "One Hop Lookups for Peer-to-Peer Overlays," in HotOS, 2003.

[18] A. Gupta, B. Liskov, and R. Rodrigues, "Efficient Routing for Peer-to-Peer Overlays.," in Proceedings of the 1st Symposium on Networked Systems Design and Implementation NSDI, 2004.

[19] J. Buford, A. Brown, and M. Kolberg, "Analysis of an Active Maintenance Algorithm for an O ( 1 ) -Hop Overlay," in IEEE Global Telecommunications, 2007, no. 1, pp. 81-86.

[20] J. Li, J. Stribling, R. Morris, and M. Kaashoek, "Bandwidthefficient management of DHT routing tables," in Proceedings of the 2nd USENIX Symposium on Networked Systems Design and Implementation, 2005, pp. 99-114.

[21] A. Brown, M. Kolberg, and J. Buford, "Chameleon: An adaptable 2-tier variable hop overlay," 6th IEEE Consum. Commun. Netw. Conf. CCNC 2009, pp. 1-6, 2009.

[22] R. S. King, "Building a Subversive Grassroots Network: How Commotion Wireless plans to enable digital communication in the face of an Internet shutdown," IEEE Spectrum, 2011. [Online]. Available: http://spectrum.ieee.org/telecom/internet/building-a-subversivegrassroots-network.

[23] X. Hong, K. Xu, and M. Gerla, "Scalable routing protocols for mobile ad hoc networks," IEEE Netw., vol. 16, no. 4, pp. 11-21, 2002.

[24] A. Boukerche, B. Turgut, N. Aydin, M. Z. Ahmad, L. Bölöni, and D. Turgut, "Routing protocols in ad hoc networks: A survey," Comput. Networks, vol. 55, no. 13, pp. 3032-3080, Sep. 2011.

[25] E. Alotaibi and B. Mukherjee, "A survey on routing algorithms for wireless Ad-Hoc and mesh networks," Comput. Networks, vol. 56, no. 2, pp. 940-965, Feb. 2012.

[26] R. Rajaraman, "Topology control and routing in ad hoc networks: A Survey," ACM SIGACT News, vol. 33, no. 2, pp. 60-73, 2002.

[27] T. Clausen and P. Jacquet, "Optimized link state routing protocol (OLSR)," RFC:3626-http://www.ietf.org/rfc/rfc3626.txt, pp. 175, 2003.

[28] J. Garcia-Luna-Aceves and M. Spohn, "Source-tree routing in wireless networks," in IEEE international Conference on Network Protocols, 1999.

[29] S. Murthy and J. J. Garcia-Luna-Aceves, "An efficient routing protocol for wireless networks," Mob. Networks Appl., vol. 1, no. 2, pp. 183-197, 1996.

[30] A. Munaretto and M. Fonseca, "Routing and quality of service support for mobile ad hoc networks," Comput. Networks, vol. 51, no. 11 , pp. 3142-3156, 2007.

[31] S. Das, C. Perkins, and E. Belding-Royer, "Ad hoc on-demand distance vector (AODV) routing," http://www.ietf.org/rfc/rfc3561.txt, 2003.
[32] D. Johnson, Y. Hu, and D. Maltz, "The Dynamic Source Routing Protocol (DSR) for Mobile Ad Hoc Networks for IPv4 (February 2007)," RFC 4728 - URL http//tools. ietf. org/html/rfc4728, 2007.

[33] D. Johnson, D. Maltz, and J. Broch, "DSR: The dynamic source routing protocol for multi-hop wireless ad hoc networks," in $\mathrm{Ad}$ hoc networking, 2001, pp. 139-172.

[34] M. Abolhasan, T. Wysocki, and E. Dutkiewicz, "A review of routing protocols for mobile ad hoc networks," Ad Hoc Networks, vol. 2, no. 1, pp. 1-22, Jan. 2004.

[35] Z. J. Haas, M. R. Pearlman, and P. Samar, "The Zone Routing Protocol (ZRP) for Ad Hoc Networks," no. draft-ietf-manet-zonezrp-04. pp. 1-11, 2002.

[36] C. Liu and J. Kaiser, "A Survey of Mobile Ad Hoc network Routing Protocols," Universität Ulm, Fakultät für Informatik. pp. 1-34, 2003.

[37] J. Xie, R. R. Talpade, A. Mcauley, and M. Liu, "AMRoute: Ad Hoc Multicast Routing Protocol,"Mob. networks Appl., pp. 429439, 2002.

[38] J. J. Garcia-Luna-Aceves and E. L. Madruga, "A multicast routing protocol for ad-hoc networks," in IEEE INFOCOM '99. $18^{\text {th }}$ Annual Joint Conference of the IEEE Computer and Communications Societies., 1999, pp. 784-792 vol.2.

[39] Sung-Ju Lee, M. Gerla, and Ching-Chuan Chiang, "On-demand multicast routing protocol," IEEE Wireless Communications and Networking Conference (WCNC), 1999, pp. 1298-1302 vol.3.

[40] J. Biswas, M. Barai, and S. K. Nandy, "Efficient hybrid multicast routing protocol for ad-hoc wireless networks," in 29th IEEE Intl. Conference on Local Computer Networks, 2004, pp. 180-187.

[41] Y.-B. Ko and N. H. Vaidya, "Geocasting in mobile ad hoc networks: location-based multicast algorithms," in Proceedings WMCSA. IEEE Workshop on Mobile Computing Systems and Applications, 1999, pp. 101-110.

[42] W. H. Liao, Y. C. Tesng, K. L. Lo, and J. . Sheu, "GeoGRID: A Geocasting Protocol for Mobile Ad Hoc Networks Based on GRID," Internet Technol., vol. 1, pp. 23-32, 2000.

[43] N. Qadri and A. Liotta, "Peer-to-Peer over Mobile Ad-Hoc Networks," in Next Generation Mobile Networks and Ubiquitous Computing, Information Science Reference, 2011, pp. 105-121.

[44] S. Lee, J. Jang, and K. Lee, "A Peer-to-Peer Search Scheme over Mobile Ad hoc Networks," in International Scientific Practical Conference., 2004.

[45] M. Conti, G. Maselli, G. Turi, and S. Giordano, "Cross-layering in mobile ad hoc network design," IEEE Comput. Soc., vol. 37 , no. 2, pp. 48-51, 2004.

[46] H. Pucha, S. Das, and Y. Hu, "Ekta: An efficient dht substrate for distributed applications in mobile ad hoc networks," $6^{\text {th }} I E E E$ workshop on mobile computing systems and applications (WMCSA), 2004.

[47] R. Hwang and C. Hoh, "Cross-layer design of P2P file sharing over mobile ad hoc networks," Telecommun. Syst., vol. 42, no. 12, pp. 47-61, Jun. 2009.

[48] N. Shah, D. Qian, and R. Wang, "MANET adaptive structured P2P overlay," Peer-to-Peer Netw. Appl., Vol. 5(2), pp. 143-160, Nov. 2012.

[49] S. a. Abid, M. Othman, and N. Shah, "3D P2P overlay over MANETs," Comput. Networks, vol. 64, pp. 89-111, May 2014.

[50] F. Delmastro, "From Pastry to CrossROAD: CROSS-layer Ring Overlay for AD hoc networks," in $3^{\text {rd }}$ IEEE Intl. Conference on Pervasive Computing and Communications Workshops, 2005.

[51] N. Shah and D. Qian, "An efficient structured P2P overlay over MANET," $9^{\text {th }}$ ACM International Workshop on Data Engineering for Wireless and Mobile Access - MobiDE '10, 2010, pp. 57-64.

[52] A. Yu and S. T. Vuong, "A DHT-based hierarchical overlay for Peer-to-Peer MMOGs over MANETs," 7th Intl. Wireless Communications and Mobile Computing Conf. , 2011.

[53] C. Cramer and T. Fuhrmann, "Performance evaluation of chord in mobile ad hoc networks," Workshop on Decentralized resource sharing in mobile computing and networking, 2006, pp. 48-53. 
[54] G. P. Millar, E. A. Panaousis, and C. Politis, "ROBUST: Reliable overlay based utilisation of services and topology for emergency MANETs," in Future Network \& Mobile Summit, 2010, pp. 1-8.

[55] G. P. Millar, E. A. Panaousis, and C. Politis, "Distributed Hash Tables for Peer-to-Peer Mobile Ad-hoc Networks with Security Extensions," J. Networks, vol. 7, no. 2, pp. 288-299, Feb. 2012.

[56] D. N. da Hora, D. F. Macedo, L. B. Oliveira, I. G. Siqueira, A. a. F. Loureiro, J. M. Nogueira, and G. Pujolle, "Enhancing peer-topeer content discovery techniques over mobile ad hoc networks," Comput. Commun., Vol. 32(13-14), pp. 1445-1459, Aug. 2009.

[57] H. Sözer, M. Tekkalmaz, and I. Korpeoglu, "A peer-to-peer file search and download protocol for wireless ad-hoc networks," Comput. Commun., vol. 32, no. 1, pp. 41-50, Elsevier, Jan. 2009.

[58] J.-C. Liang, J.-C. Chen, and T. Zhang, "An adaptive lowoverhead resource discovery protocol for mobile ad-hoc networks," Wirel. Networks, Vol. 17(2), pp. 437-452, Nov. 2010.

[59] M. C. Castro, E. Villanueva, I. Ruiz, S. Sargento, and A. J. Kassler, "Performance Evaluation of Structured P2P over Wireless Multi-hop Networks," in $2^{\text {nd }}$ International Conference on Sensor Technologies and Applications, 2008, pp. 796-801.

[60] J. Mei, H. Ji, and T. Li, "Cross-layer optimized Chord protocol for separated ring convergence in MANET," J. China Univ. Posts Telecommun., vol. 16, no. 4, pp. 84-90, Aug. 2009.

[61] M. Lee and A. Sivasubramaniam, "Efficient peer-to-peer information sharing over mobile ad hoc networks," in MobEAII, 2004 .

[62] S. Burresi, C. Canali, M. E. Renda, and P. Santi, "M ESH C HORD : A Location-Aware, Cross-Layer Specialization of Chord for Wireless Mesh Networks," in $6^{\text {th }}$ IEEE Intl. Conf. on Pervasive Computing and Communications, 2008, pp. 206-212.

[63] R. Kummer, P. Kropf, and P. Felber, "Distributed lookup in structured peer-to-peer ad-hoc networks," in On the Move to Meaningful Internet Systems, Springer, 2006, pp. 1541-1554.

[64] S. G. Fantar and H. Youssef, "Locality-aware Chord over Mobile Ad Hoc Networks," in Global Information Infrastructure Symposium, 2009, pp. 1-6.

[65] G. Peng, S. Li, H. Jin, and T. Ma, "M-CAN: a lookup protocol for mobile peer-to-peer environment," in Parallel Architectures, Algorithms and Networks, 2004, pp. 544- 549.

[66] M. Li, E. Chen, and P. Sheu, "A chord-based novel mobile peerto-peer file sharing protocol," in 8th Asia-Pacific Web Conference, 2006, pp. 806-811.

[67] M. Thaalbi, A. Meddahi, T. Bejaoui, and N. Tabbane, "An enhanced chord-based P2P lookup protocol for mobile Ad hoc networks," in Wireless Days (WD) IFIP, 2011.

[68] M. Thaalbi, N. Tabbane, T. Bejaoui, and A. Meddahi, "Enhanced Backtracking Chord protocol for mobile Ad hoc networks," in International Conference on Communications and Information Technology (ICCIT), 2012, pp. 191-195.

[69] C. Cramer and T. Fuhrmann, "Proximity Neighbor Selection for a DHT in Wireless Multi-Hop Networks," $5^{\text {th }}$ IEEE International Conference on Peer-to-Peer Computing, 2005, pp. 3-10.
[70] M. Shin and W. A. Arbaugh, "Efficient peer-to-peer lookup in multi-hop wireless networks," KSII Trans. Internet Inf. Syst., vol. 3, no. 1, pp. 5-25, 2009.

[71] M. Caesar, M. Castro, E. Nightingale, G. O'Shea, and A. Rowstron, "Virtual ring routing: network routing inspired by DHTs," Conf. Applications, technologies, architectures, and protocols for computer communications, 2006, pp. 351-362.

[72] T. Fuhrmann, P. Di, K. Kutzner, and C. Cramer, "Pushing chord into the underlay: Scalable routing for hybrid manets," Univ. Karlsruhe (TH), Fak. für Inform. Tech. Rep., 2006.

[73] Y. Hu, S. Das, and H. Pucha, "Exploiting the Synergy between Peer-to-Peer and Mobile Ad Hoc Networks.," in HotOS Ninth Workshop on Hot Topics in Operating Systems, 2003.

[74] T. Zahn and J. Schiller, "MADPastry: A DHT substrate for practicably sized MANETs," Proc. ASWN, 2005.

[75] Q. Meng and H. Ji, "MA-Chord: A New Approach for Mobile Ad Hoc Network with DHT Based Unicast Scheme," in International Conference on Wireless Communications, Networking and Mobile Computing, 2007, pp. 1533-1536.

[76] A. Carneiro Viana, M. Dias de Amorim, S. Fdida, and J. Ferreira de Rezende, "An Underlay Strategy for Indirect Routing," Wirel. Networks, vol. 10, no. 6, pp. 747-758, Nov. 2004.

[77] J. I. Alvarez-Hamelin, A. C. Viana, and M. D. de Amorim, "DHT-based Funcionalities Using Hypercubes," in IFIP 19th World Computer Congress, TC-6, IFIP Interactive Conference on Ad-Hoc Networking, 2006, vol. 212, pp. 157-176.

[78] J. Eriksson, M. Faloutsos, and S. V. Krishnamurthy, "DART: Dynamic Address RouTing for Scalable Ad Hoc and Mesh Networks," IEEE/ACM Trans. Netw., vol. 15, no. 1, pp. 119-132, Feb. 2007.

[79] M. Caleffi, G. Ferraiuolo, and L. Paura, "Augmented tree-based routing protocol for scalable ad hoc networks," Intl. Conf. on Mobile Adhoc and Sensor Systems, IEEE, 2007, no. 2005093248.

[80] M. Caleffi and L. Paura, "P2P over MANET: Indirect tree-based routing," IEEE Intl. Conference on Pervasive Computing and Communications, 2009, pp. 1-5.

[81] J. J. Garcia-Luna-Aceves and D. Sampath, "Scalable integrated routing using prefix labels and distributed hash tables for MANETs," IEEE 6th International Conference on Mobile Adhoc and Sensor Systems, MASS '09, 2009, pp. 188-198.

[82] B. Zhao, Y. Wen, and H. Zhao, "KDSR: An Efficient DHTBased Routing Protocol for Mobile Ad Hoc Networks," $9^{\text {th }}$ Intl. Conference on Hybrid Intelligent Systems, 2009, pp. 245-249.

[83] S. a. Abid, M. Othman, and N. Shah, "Exploiting 3D structure for scalable routing in MANETs," IEEE Commun. Lett., vol. 17, no. 11, pp. 2056-2059, 2013.

[84] M. Al Mojamed, M. Kolberg; Design and evaluation of a peer-topeer MANET crosslayer approach: OneHopOverlay4MANET; Peer-to-Peer Networking and Applications Journal, Springer, 2015, DOI: 10.1007/s12083-015-0413-4. 